\title{
Functional Properties of Parietal Visual Neurons: Radial Organization of Directionalities Within the Visual Field
}

\author{
M. A. Steinmetz, B. C. Motter, C. J. Duffy, and V. B. Mountcastle \\ The Bard Laboratories of Neurophysiology, Department of Neuroscience, The Johns Hopkins University School of \\ Medicine, Baltimore, Maryland 21205
}

\begin{abstract}
Parietal visual neurons (PVNs) were studied in waking monkeys as they executed a simple fixation-detection task. Test visual stimuli of varied direction, speed, and extent were presented during the fixation period; these stimuli did not control behavior. Most PVNs subtend large, bilateral receptive fields and are exquisitely sensitive to stimulus motion and direction but insensitive to stimulus speed. The directional preferences of PVNs along meridians are opponently organized, with the preferred directions pointing either inward toward or outward away from the fixation point. Evidence presented in the preceding paper (Motter et al., 1987) indicates that opponent directionality along a single meridian is produced by a feed-forward inhibition of $20^{\circ}-30^{\circ} \mathrm{spa-}$ tial extent. The observations fit a double-Gaussian model of superimposed but unequal excitatory and inhibitory receptive fields: When the former is larger, inward directionality results; when smaller, outward directionality results. We examine here the distribution of the meridional directional preferences in the visual field. Tests showed that opponent organization is not produced by differences in local directional properties in different parts of the receptive field. The distribution of response intensities from one meridian to another is adequately described by a sine wave function. These data indicate a best radial direction for each neuron with a broad distribution of response intensities over successive meridians. Thus, any single PVN, with rare exceptions, cannot signal radial stimulus direction precisely. We then determined how accurately the population response predicted radial stimulus direction by the application of a linear vector summation model. The resulting population vector varied from stimulus direction by an average of $9^{\circ}$. Whether or not the perception of the direction of motion depends upon a population vector remains uncertain.

PVNs are especially sensitive to object movement in the visual surround, particularly in the periphery of the visual field. This, combined with their large receptive fields and their wide but flat sensitivity to stimulus speed, makes them especially sensitive to optic flow. This is discussed in relation to the role of the parietal visual system in the visual guidance of projected movements of the arm and hand, in the guidance of locomotion, and in evoking the illusion of vection.
\end{abstract}

\footnotetext{
Received Mar. 13, 1986; revised May 23, 1986; accepted June 5, 1986.

The research described in this paper was supported by Grant 5 R01 EY03168 from the National Institutes of Health, United States Public Health Service.

Correspondence should be addressed to V. B. Mountcastle at the above address.

Copyright (C) 1987 Society for Neuroscience 0270-6474/87/010177-15\$02.00/0
}

The preceding paper (Motter et al., 1987) gives a description of the general properties of parietal visual neurons (PVNs) and their sensitivities to stimulus motion, direction, and speed along single meridians through the frontoparallel plane. The most unusual properties of the majority of PVNs are the bilateral distributions of their receptive fields and the "opponent" organization of the directional properties within them. That is, many PVNs respond to stimuli moving radially either inward toward or outward away from the point of fixation, such that along a single meridian the directional selection changes, sometimes abruptly, as the edge of the stimulus crosses the central line of gaze. Evidence was presented that this property is determined by a feed-forward inhibitory process acting in the direction of stimulus movement. This mechanism extends from the local edge of the moving stimulus for distances of $10^{\circ}-20^{\circ}$ ahead of it into the receptive field. The result is that when stimuli traverse large extents of visual space, movement through one region conditions the response to subsequent stimulus motion through other regions of the visual field. The inhibitory action is thought to depend upon a differential distribution of overlapping excitatory and inhibitory receptive fields, an asymmetric distribution of inhibition, or both.

We observed in these experiments that opponent directionality is rarely limited to a single meridian and may exist along each of the 4 meridians tested. However, the intensity of response for stimuli moving in the preferred radial direction, i.e., inward or outward, is seldom equal for all radial axes. Most often, the evoked discharge rate varies as a smooth and continuous function of the angular position of the meridian tested, relative to the central line of gaze, in the frontoparallel plane. The analyses of these data are presented. We then consider the results with reference to several propositions: (1) the mechanism of meridional selectivity in the visual field, (2) whether there is a reasonably precise signal of stimulus direction embedded in the PVN population pattern of discharge, and (3) what role the parietal visual system plays in the perception of self-motion, in the visual guidance of locomotion, and in the visual guidance of projected movements of the arm and hand.

\section{Materials and Methods}

The behavioral and electrophysiological methods, animal training procedures, and details of the visual stimuli are described in the preceding paper (Motter et al., 1987). Briefly, macaque monkeys were trained to achieve and maintain fixation of a $0.2^{\circ}-0.3^{\circ}$ target light for periods of $1-5 \mathrm{sec}$ and to detect its dimming to receive a liquid reward. Visual test stimuli consisting of $10^{\circ} \times 10^{\circ}$ squares of which light were projected and moved across a tangent screen during the fixation period. Steady eye position was verified by electro-oculographic recording using $\mathrm{Ag}-$ $\mathrm{AgCl}$ electrodes surgically implanted in the orbital rims. The action 


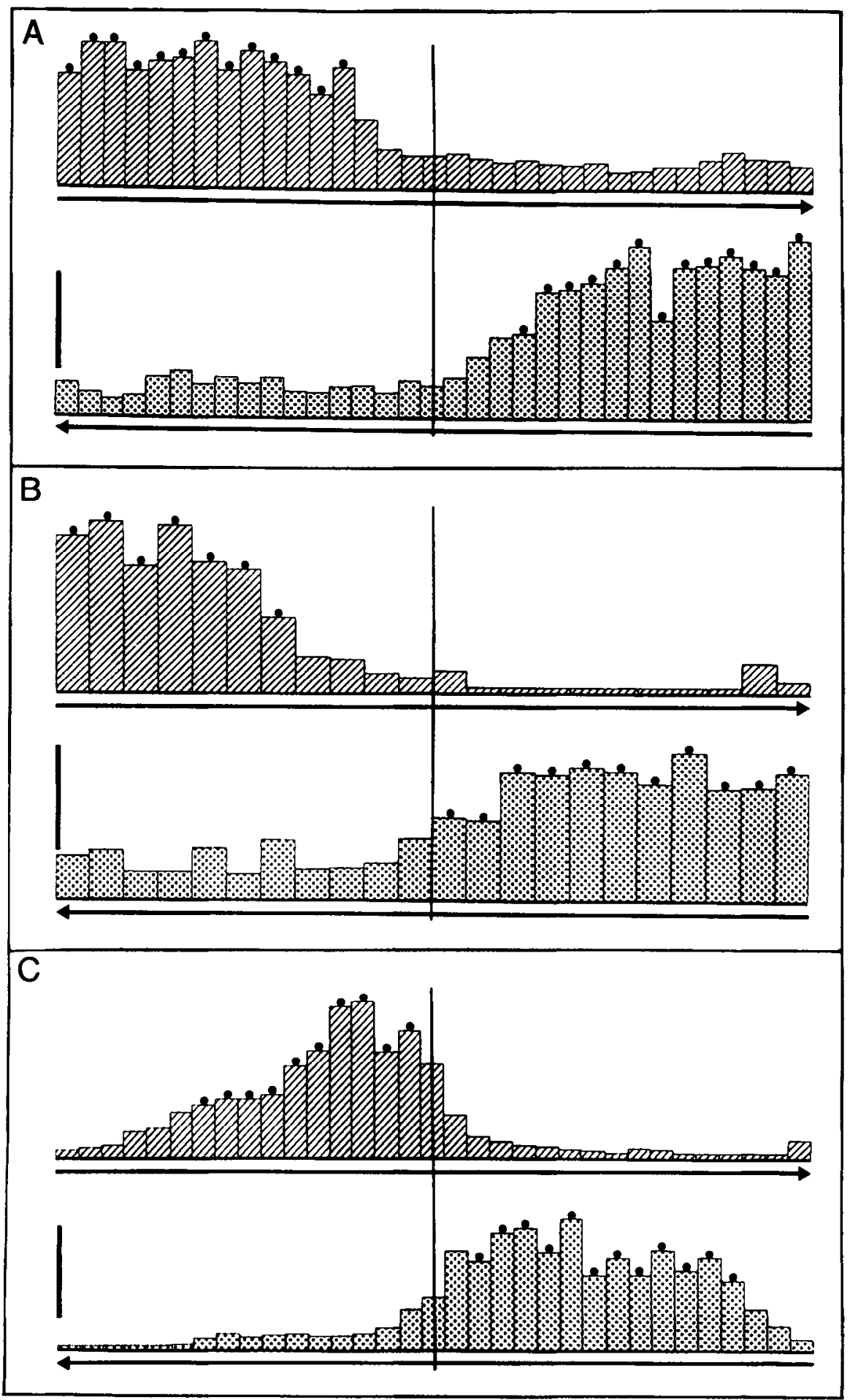

Figure 1. Peristimulus time histograms of the responses of 3 different parietal visual neurons $(A-C)$ to opposite directions of stimulus motion, indicated by arrows, along single meridians. Solid circles indicate histogram cells in which responses were significantly greater than those observed for the opposite direction of motion through the same zones of space $(t$ test, $p<$ 0.05 ). The stimuli moved for $100^{\circ}$ along meridians centered at the point of fixation, indicated by the long vertical bars. Calibration bars at the left indicate response frequencies of 100 impulses/sec. Stimulus speed, $60 \% \sec (A$ and $C), 90 \%$ $\sec (B)$.

potentials of individual PVNs were identified and isolated using conventional electrophysiological techniques. The optimal stimulus velocity was determined from a preliminary series of trials that spanned a range of $30^{\circ}-600^{\circ} / \mathrm{sec}$. The stimulus velocity that produced the maximum rate of discharge was then used for subsequent tests. It was $60^{\circ}$ or $90 \% \mathrm{sec}$ for most neurons.

Two types of stimulus sets were used. Each consisted of stimulus motion in 8 equally spaced directions in the frontoparallel plane. The first set employed stimulus movement of $100^{\circ}$ in extent centered on the point of fixation, moving in each of the 2 directions along the vertical, horizontal, and 2 equally spaced diagonal meridians. Local receptive field properties were tested with a second set of stimuli moved only $10^{\circ}$ or $20^{\circ}$ in each of 8 equally spaced directions in the circular dimension, centered either over the point of fixation or at any location at $10^{\circ}$ intervals along any of the 4 meridians ("local rotate runs," see Fig. 3).

The optimal direction of stimulus movement for each PVN was estimated by periodic regression (Batschelet, 1981), using the method of least squares to fit a sine wave of the form

$$
y=b_{0}+b_{1} \sin (\theta)+b_{2} \cos (\theta)
$$

or, in terms of the preferred direction,

$$
y=b_{0}+c_{1} \cos \left(\theta-\theta_{0}\right)
$$

with 

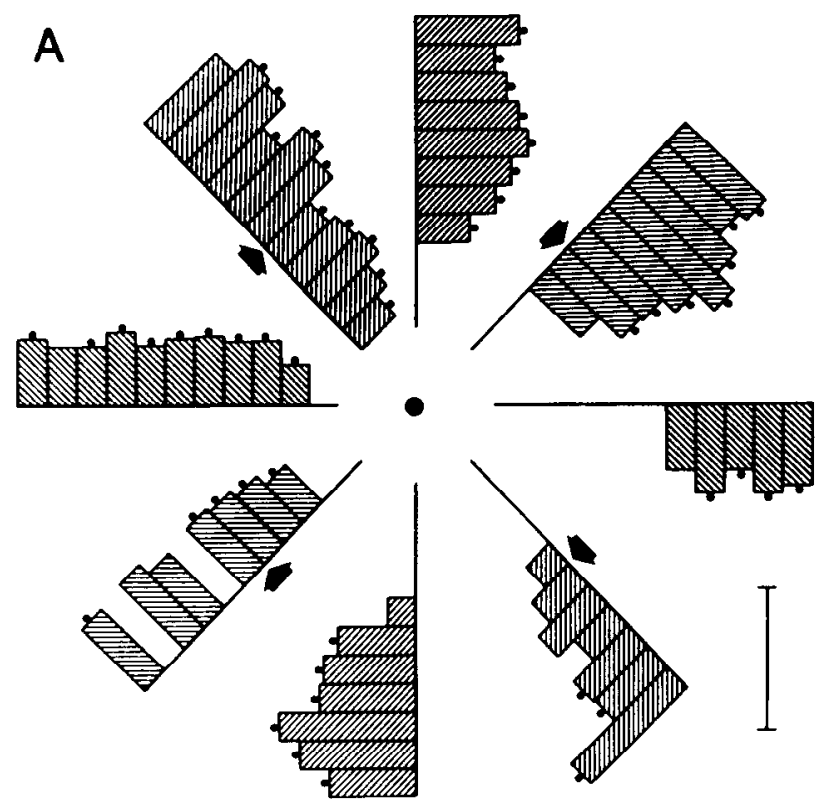

Figure 2. Radially oriented histograms of the responses of 2 parietal visual neurons $(A$ and $B)$ to 8 directions of motion. Only those histogram bins in which the discharge rate is significantly different from the ongoing background activity $(t$ test, $p<0.05)$ are shown. Bins shaded with diagonal cross-hatching are responses to inward stimulus motion; stippled bins are responses to outward motion away from the point of fixation (large central dot), along the same meridian. Bins marked with solid circles indicate response rates significantly different from those evoked by stimuli moving in the opposite direction through the same zones of space (paired $t$ test, $p<0.05$ ). Stimulus velocity was $90^{\circ}$ and $60^{\circ} /$ sec in $A$ and $B$, respectively. The spatial extent of each stimulus movement was $100^{\circ}$, centered on the point of fixation. The histograms have been separated for $20^{\circ}$ about the fixation point for ease of viewing. Calibration bars, 100 impulses/sec. Arrows in $A$ indicate the spatial locations of tests of local directionality shown in Figure 3.

$$
c_{1}=\sqrt{b_{1}^{2}+b_{2}^{2}}
$$

where

$y=$ the PVN discharge rate for stimulus movement in direction $\theta$

$b_{0}, b_{1}$, and $b_{2}=$ regression coefficients,

$c_{1}=$ maximum increase from the average discharge rate,

$\theta=$ direction of stimulus motion,

$\theta_{0}$ - the "best" or "preferred" direction of motion.

Using least-squares methods to fit the coefficients $b_{0}, b_{1}$, and $b_{2}$ in equation (1) and substituting them into equations (2) and (3), the "preferred" direction $\left(\theta_{0}\right)$ can be calculated using the following correction for quadrant:

$\theta_{0}^{\prime}=\tan ^{-1}\left(b_{1} / b_{2}\right)$,

$\theta_{0}=\theta_{0}^{\prime}, \quad$ if $b_{1}>0, b_{2}>0$

$\theta_{0}=\theta_{0}^{\prime}+180^{\circ}, \quad$ if $b_{2}<0$

$\theta_{0}=\theta_{0}^{\prime}+360^{\circ}, \quad$ if $b_{1}<0, b_{2}>0$.

The correlation coefficient can be calculated as

$$
r^{2}=4\left(b_{1}{ }^{2}+b_{0}{ }^{2}\right) /\left(y-b_{0}\right)^{2}
$$

An index of circular tuning was calculated from the responses to the 8 directions of stimulus motion; it is the width in degrees in the circular dimension in which the increases in the PVN discharge rate over the rate of background activity exceed $50 \%$ of the maximum increase above the background rate observed. Linear interpolation between adjacent points was used to determine the intersections of the data curves with the $50 \%$ response level.

\section{Results}

The observations and analyses described below were made on the basis of the 188 PVNs described in the previous paper (Motter et al., 1987). One hundred thirty-three of those neurons qualified for the present analysis on the basis of the completeness of study and the statistical validity of their responses. Each

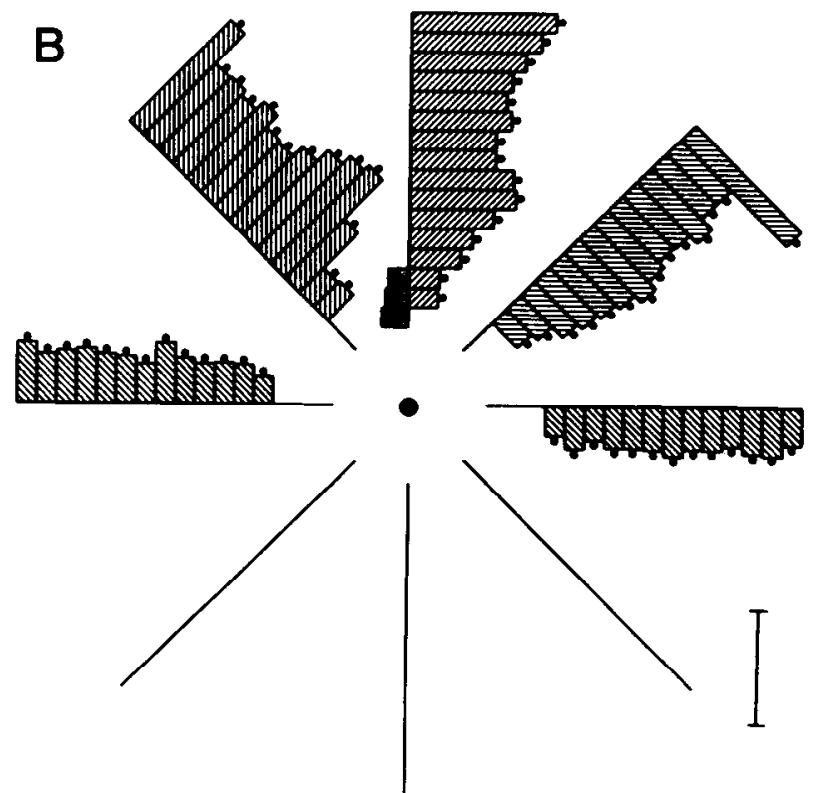

neuron was tested by light stimuli that moved in either direction for $100^{\circ}$ along each of 4 evenly spaced meridians; the lengths of stimulus movements were centered at the point of fixation. In addition, 269 tests of directionality within local regions of the receptive fields were made on 92 neurons of this population, in the manner described above.

The response histograms for 3 PVNs shown in Figure 1 demonstrate opponent organization of directionality for stimuli moved in each of the 2 directions along a single meridian. Opponent directionality in PVNs is seldom limited to a single meridian; for many neurons it occurs along several or all the meridians tested. The variations in directionality and response intensity such as those shown in Figure $2 A$ support the idea that for almost all PVNs the directional properties differ for different meridians. The neuron of Figure $2 A$ responded as the stimulus moved inward toward the fixation point along each of the 8 directions tested along 4 meridians in the plane of the tangent screen; the cell did not respond at all during stimulus movements outward away from the point of fixation. Thus, the true angular directional selectivity appeared to change regularly from meridian to meridian. The intensities and durations of the responses to inwardly moving stimuli did vary from one meridian to the next, producing the moderate circular asymmetry illustrated by the histograms of Figure $2 A$. The organization of the radial directionalities in the visual field is analyzed in detail below. The neuron of Figure $2 B$ was activated from a receptive field that filled the upper half of the visual field. The axial directional selection was inward and changed in true angular direction from meridian to meridian, producing an opponent directionality along the horizontal meridian. The spectrum of receptive field sizes included those of neurons with opponent 


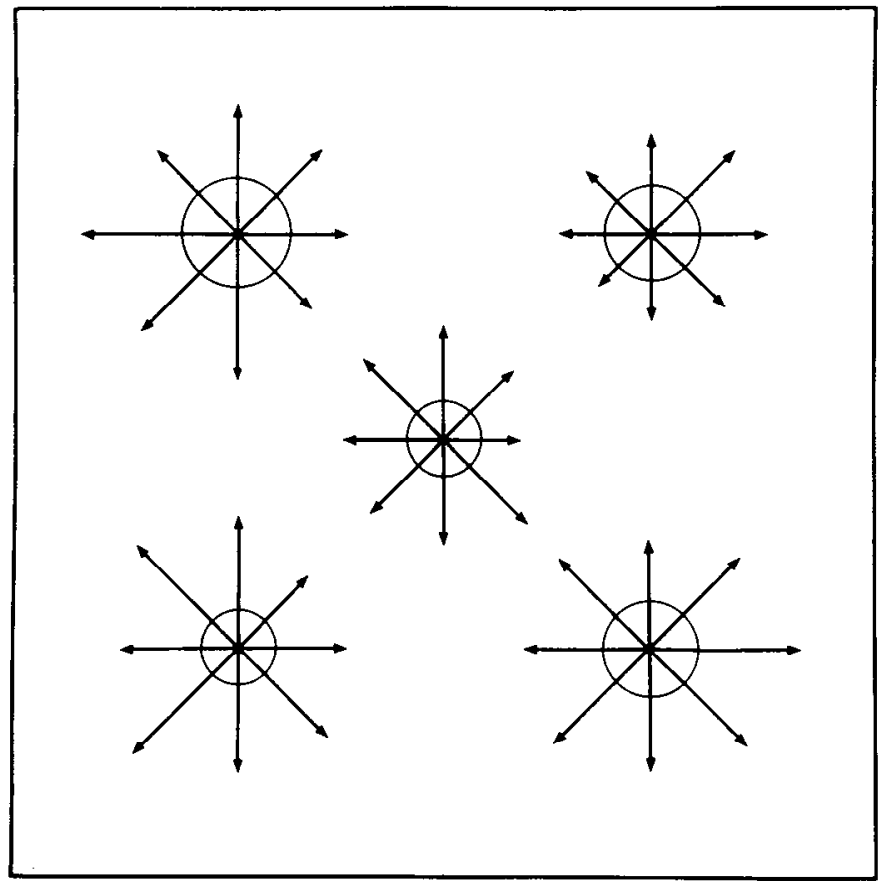

Figure 3. Results of tests for local directionality for the parietal visual neuron of Figure $2 A$ at the point of fixation and at 4 positions $20^{\circ}$ from the point of fixation along the diagonal meridians (see arrows in Fig. $2 A$ ). The response to each stimulus movement is represented by a vector whose length is proportional to the mean rate of discharge and whose direction indicates the direction of stimulus motion. Circles indicate rate of ongoing discharge in the absence of a visual stimulus. Radius of the circle at upper left corresponds to a discharge rate of 30 impulses/ sec.

directionality along a single responsive meridian. For most PVNs the circular extent of opponency lay between 2 extremes, from those neurons activated from only a single meridian to the one illustrated in Figure $2 A$. The majority of PVNs ( 80 of 133) showed some degree of opponent organization, including both those for which the field includes the foveal region and those in which that region is spared.

\section{What mechanisms account for changes in the axial selectivity of PVNs?}

The evidence presented in the preceding paper (Motter et al., 1987) indicates that the directional preference of PVNs along a single meridian is produced by an inhibition that acts from the edge for a wide distance ahead of a stimulus moving in the null direction. Here, we address the question, What mechanisms produce the change in true angle of the directional preference that accompanies each angular shift in the meridian tested; i.e., the virtual absence of axial selectivity? It is obvious that while the axial directionalities of Figure $2 A$ are strongly inward for each axis tested, the angle of those directionalities in the frontoparallel plane of the tangent screen varies over the full range of $360^{\circ}$.

The first possibility we consider is that a local or minute directional property exists throughout the receptive field of a cell like that of Figure $2 A$ and that the true angle of the local directional preference shifts progressively from one location to another in the receptive field, producing patterns of opponency like those of Figure $2 A$ and Figures 3, 4, and 5, $A-C$, of the
Table 1. Comparison of the directionality observed in the full-field and local tests for 13 PVNs tested in 4 or more local regions of the receptive field

\begin{tabular}{|c|c|c|c|c|c|c|}
\hline \multirow[b]{2}{*}{$\begin{array}{l}\text { Full-field } \\
\text { tests }\end{array}$} & \multicolumn{5}{|c|}{ Local tests } & \multirow[b]{2}{*}{ Totals } \\
\hline & Inward & $\begin{array}{l}\text { Out- } \\
\text { ward }\end{array}$ & $\begin{array}{l}\text { Non- } \\
\text { direc- } \\
\text { tional }\end{array}$ & $\begin{array}{l}\text { Un- } \\
\text { re- } \\
\text { spon- } \\
\text { sive }\end{array}$ & Other & \\
\hline Inward & 4 & 0 & 14 & 0 & 6 & 24 \\
\hline Outward & 0 & 0 & 2 & 0 & 0 & 2 \\
\hline Nondirectional & 1 & 0 & 4 & 2 & 2 & 9 \\
\hline Unresponsive & 2 & 0 & 9 & 2 & 5 & 18 \\
\hline Totals & 7 & 0 & 29 & 4 & 13 & 53 \\
\hline
\end{tabular}

The 53 local tests were labeled inward or outward if the preferred direction was within $22.5^{\circ}$ of the 2 directions along the meridian on which the test was made, and "other" if it was outside that range. The full-field tests were labeled inward, outward, nondirectional, or unresponsive for each of the 8 directions tested.

preceding paper (Motter et al., 1987). We have tested this proposition by examining local directional properties and axial selection in different parts of the receptive fields of PVNs, using the "local rotate" stimulus set described in Materials and Methods. (The set consists of stimuli moving at $60^{\circ}$ or $90^{\circ} / \mathrm{sec}$, of $20^{\circ}$ movement length, delivered at $45^{\circ}$ intervals of direction around a circle centered at a selected point in the receptive field.) We tested 269 zones in the receptive fields of 92 PVNs. Of these, less than half (129 of 269) showed any significant axial selectivity by sine wave regression analysis. We then analyzed the results for a subset of 13 PVNs for each of which 4 or more local sites were tested, usually at locations $20^{\circ}$ from the fixation point along the diagonal meridians. We first compared any axial directionality demonstrated with stimuli that moved for $20^{\circ}$ with that observed in the same $20^{\circ}$ segment of the meridian by full-field stimuli of $100^{\circ}$ movement. We then determined whether any axial selectivity could be demonstrated in each local region and, if so, whether any axial selectivity observed differed in different parts of the receptive field. The result of this experiment for the neuron of Figure $2 A$ is shown in Figure 3. The responses at each location to stimuli moving in the 8 directions tested are plotted as vectors whose lengths are proportional to the average evoked response rate and whose directions are those of stimulus motion. The local tests of Figure 3 were made at the receptive field locations marked by arrows in Figure $2 A$. The trigonometric moment method of Mardia (1972) was used to determine if a significant axial selection existed at any of the local test positions. The mean resultant length, a measure of dispersion, was calculated from these data; in each of the 5 cases this measure did not differ significantly from zero by the Rayleigh test (Mardia, 1972). These results indicate that no local axial selectivity existed within the receptive field of the neuron of Figure $2 A$, and thus that the all-inward directionality shown for the $8 \mathrm{di}$ rections tested along the 4 meridians with full-field stimuli must be produced by other than local directional mechanisms.

A total of 53 local positions in the subset of 13 neurons described above was tested in this way, at positions that could be compared with the results obtained with full-field stimuli. The results of the comparison are given in Table 1 . Of the 26 local sectors lying on meridians for which cells were shown to be directionally selective by full-field stimuli, only 4 were directionally selective in the same direction $\left( \pm 22.5^{\circ}\right)$ by the local 


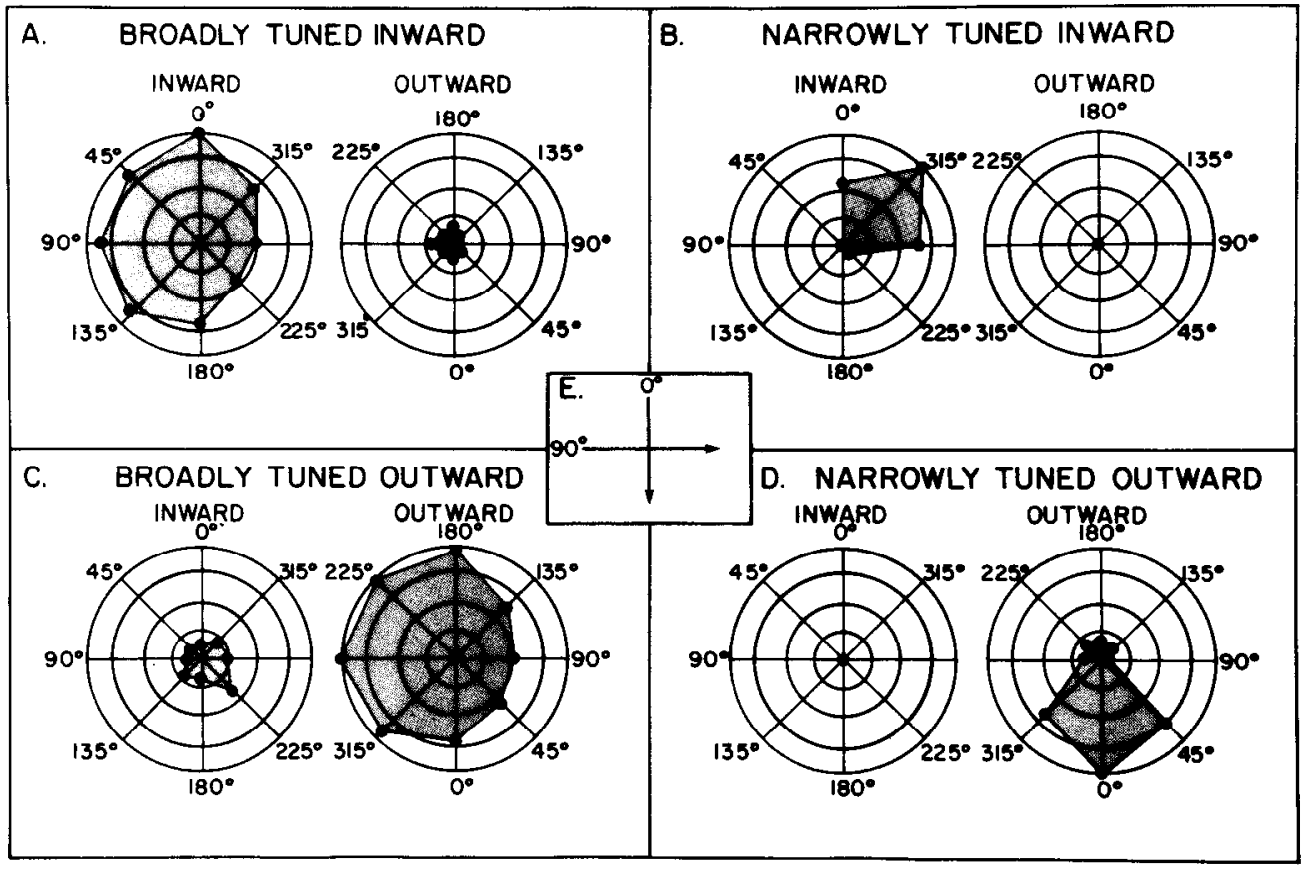

Figure 4. Polar plots of the average discharge rate for four PVNs $(A-D)$ during the inward and outward halves of the "full-field" tests of directionality. Stimuli traversed $100^{\circ}$ along each meridian, centered on the point of fixation. Responses are given as percentages of the maximum rate for each neuron. Plots are rotated so that the directional axes correspond with spatial locations in the frontoparallel plane. Actual directions of the stimulus motion are indicated in each plot, with $0^{\circ}$ indicating a downward motion in the frontoparallel plane and with directional labels increasing in the counterclockwise direction (see inset $E$ ). tests. The outcomes of the comparison of the 2 sets are shown by the numbers in the cells of Table 1. The most common outcome was that a local zone, shown to be directional in the full-field test along that particular meridian, was nondirectional in the local test. Of all the possible outcomes, only 10 of 53 are congruent between the 2 sets of data.

Several possible explanations of the directional organization within the receptive fields of PVNs can, we believe, be dismissed on the basis of present evidence or because of the rarity of occurrence of the properties required. For example, it is possible that the convergence from the 2 hemispheres, obviously required to compose the receptive fields of PVNs, is arranged so that neurons from the 2 hemispheres with opposing directionalities toward or away from a major axis like the vertical meridian converge upon the same PVN. Such a convergence could produce an opponent directionality along the horizontal meridian and other horizontal axes as well. This explanation is unlikely because we have observed only a single instance of such a pattern of directionality for a PVN (Motter and Mountcastle, 1981, Fig. 17), because examples of opposing local directionalities in opposite halves of receptive fields have not been observed, and because no explanation is provided for opponent organization along both the horizontal and vertical meridians in the same cell.

Another hypothesis is that PVNs have a broadly tuned but uniform directionality within their receptive fields and that directionality appears to be organized in the opponent pattern only because we have used stimuli that moved along meridians. We think this explanation unlikely because we have not observed a single PVN with a giant receptive field and opponent organization of directionality that showed such a uniform directionality when tested with local stimuli. Moreover, the majority of such local zones show no significant directionality at all, even when responses to local stimuli are vigorous.

The directional properties of 80 of the 133 neurons in the population of PVNs analyzed were organized in an opponent manner, like those of Figures 1 and 2. The remaining 53 neurons were related to small foveal receptive fields, to fields restricted to one half of a single meridian, or to fields that occupied less than $180^{\circ}$ of the visual field. Many of these showed directional selectivity along a portion of a single meridian, but nonc, of course, could show the opponent organization we wished to analyze.

In summary, the majority of PVNs display a directional selectivity along meridians through their large and frequently bilaterally symmetrical receptive fields. These directional preferences are frequently arranged along each meridian in opponent fashion, either inward or outward with respect to the point of fixation. The angle of the preferred axial direction in the frontoparallel plane thus changes with the angular inclination of the meridian tested and, for many cells, varies over the full range of $360^{\circ}$. Tests of local positions within receptive fields show that at the majority of locations no axial selectivity exists, and, when it can be demonstrated, it rarely accounts for the patterns of axial specificity and selectivity observed with full-field stimuli. We infer by exclusion that the difference in the directionality of PVNs along different meridians, like that along a single one, is produced by the feed-forward inhibitory effect described in the preceding paper (Motter et al., 1987). It is presently best understood, we believe, in terms of the double-Gaussian model described and illustrated in Figure 16 of that paper.

\section{Organization of the meridional directionalities of PVNS}

Although PVNs may respond preferentially to inward or outward motion along all meridians, the intensity of response indexed by either the mean or the peak rate of neuronal discharge varies smoothly from one meridian to another. These differences in the intensities of responses to stimuli moving along different meridians in the frontoparallel plane suggest that PVNs may provide reliable signals of the direction of motion in the visual surround relative to the central line of gaze. Extremes in the nature of the response distribution from meridian to meridian in the "circular" dimension, observed in the population of PVNs, are shown in Figures 4 and 5, where the average rates of dis- 


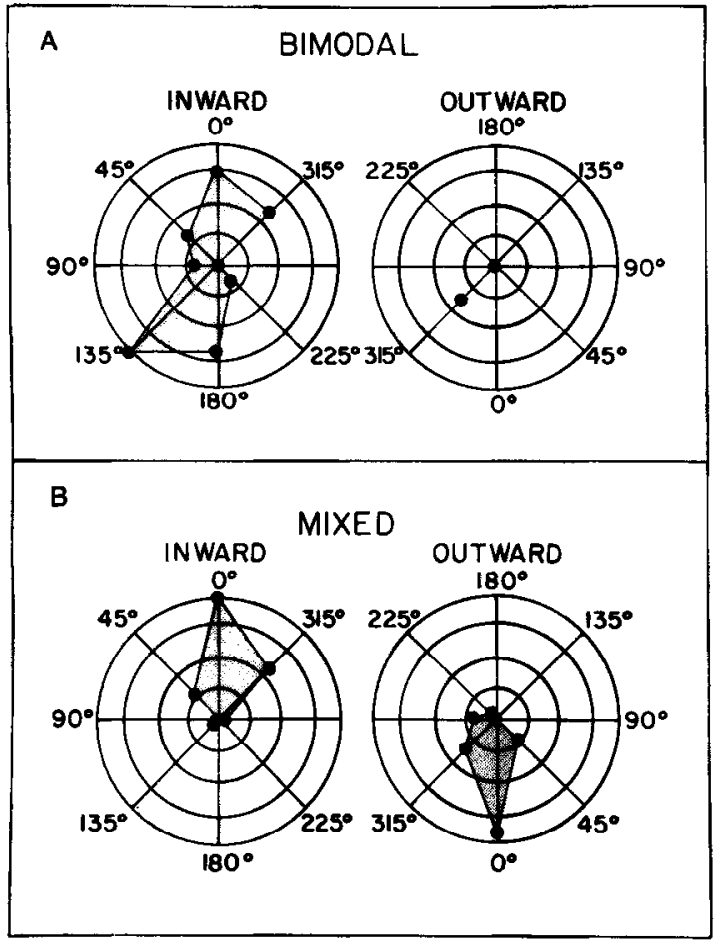

Figure 5. Polar plots showing 2 response patterns less commonly observed in PVNs. See text and legend to Figure 4 for description.

charge during inward and outward stimulus movements are plotted separately as functions of the direction of motion along the meridians tested. Figure $4, A$ and $B$, presents examples of broad and narrow tuning for neurons with inward axial directionality. Figure $4 A$ illustrates a nearly balanced opponency in which the intensity of response is almost the same for 6 of the 8 directions of inward motion. Figure $4 B$ illustrates the results for a PVN whose responses are narrowly tuned for a particular direction of motion. Figure 4, $C$ and $D$, shows similar examples of PVNs with outward axial directionalities.

Examples of other, less common response patterns are shown in Figure 5. The neuron of Figure $5 A$ had a "bimodal" distribution of axial response intensities, with opponent inward directionality. Figure $5 B$ shows results for a $\mathrm{PVN}$ in which the directionality appears to be uniform along a single axis. This cell responded preferentially to the same direction of motion on the opposite sides of the fixation point. The majority of such neurons had receptive fields that included the point of fixation and responded to inward stimulus motion over spatial extents that overlapped the fixation point, thus producing an outward directionality on the other side of the line of gaze. Table 2 summarizes our findings: (1) The majority (104 of 133) of the PVNs analyzed have a continuous distribution of response intensity in the "circular" dimension of the frontoparallel plane, with an optimal or "best" direction; (2) the majority of PVNs (85 of 133) respond preferentially to one radial direction, either inward or outward relative to the central line of gaze.

It is possible to determine the optimal radial direction from the responses of PVNs by a number of methods. We present below the results obtained using a sinusoidal regression analysis, as described in Materials and Methods. Identical results are obtained if the trigonometric moment method of Mardia (1972)

\begin{tabular}{lccc}
\hline $\begin{array}{l}\text { Table 2. Summary of radial directional preferences based on the full- } \\
\text { field tests }\end{array}$ \\
$\begin{array}{l}\text { Radial } \\
\text { direction } \\
\text { preferred }\end{array}$ & Unimodal & Bimodal & Totals \\
\hline Inward & 54 & 12 & 66 \\
Outward & 15 & 4 & 19 \\
Mixed & 35 & 13 & 48 \\
Totals & 104 & 29 & 133 \\
\hline
\end{tabular}

Neurons with single peaks in the distribution of directional response intensities were classified as unimodal and those with 2 peaks as bimodal. See Figures 4 and 5.4 for examples.

is used. Figure 6 illustrates the results of the sine wave regression analysis for a PVN that responded preferentially to inwardly moving stimuli. The PST histograms at the top of the figure show the time course of the response to stimuli moving in each of the 8 directions of motion tested. The mean and peak rates of discharge are plotted separately below for the inward and outward motions relative to the point of fixation, as functions of the directions of stimulus motion along different meridional axes. The dotted lines in these graphs indicate sine waves fitted to the data by the least-squares method. The responses of this PVN are adequately described by a sinusoidal function with correlation coefficients of 0.95 for mean responses and 0.94 for peak responses. The most effective direction of stimulus motion is defined by the peak of the sine wave; for this neuron it is $9^{\circ}$ counterclockwise from the downward direction along the vertical meridian. The best direction is virtually the same for mean and peak responses.

The results of sinusoidal regression analyses of the responses of 2 other PVNs are shown in Figure 7. The neuron of Figure $7 A$ responded preferentially to inward stimulus motion, with a best direction of $8^{\circ}$ counterclockwise from the vertical. The neuron of Figure $7 B$ responded preferentially to outward stimulus motion, with a best direction of $191^{\circ}$. For this neuron, weak responses were evoked by inward motion as the leading edge of the stimulus approached and crossed the point of fixation. The close fit of these responses to a sine wave is shown by the corresponding $r^{2}$ values of 0.97 and 0.96 .

The histogram of Figure 8 plots the $r^{2}$ values for the sinusoidal regression analysis of all of the neurons in our population with unimodal "circular" distributions of responses to either inward or outward stimulus motion. The responses for the majority (76\%) of these neurons fit sinusoidal functions reasonably well, having $r^{2}$ values $>0.70$. There are 2 reasons for a lack of sinusoidal fit, when it occurs. Rarely $(n=3)$, the responses are nearly equal for all radial directions of stimulus motion tested, like those of the neuron of Figure $9 A$. This PVN showed a strong preference for inward over outward motion but showed no preference for one or another meridian. For a few other cells, the distribution of response intensities among meridians is very narrowly tuned; an example is given in Figure $9 B$. The optimal direction for this cell was outward from the point of fixation. Neurons of this latter type, which show the most precise axial selection of all, are included in the population analysis described below despite the lack of sinusoidal variation of their responses.

Figure 10 gives the distribution of preferred axial selections for all neurons in our population with unimodal "circular" dis- 

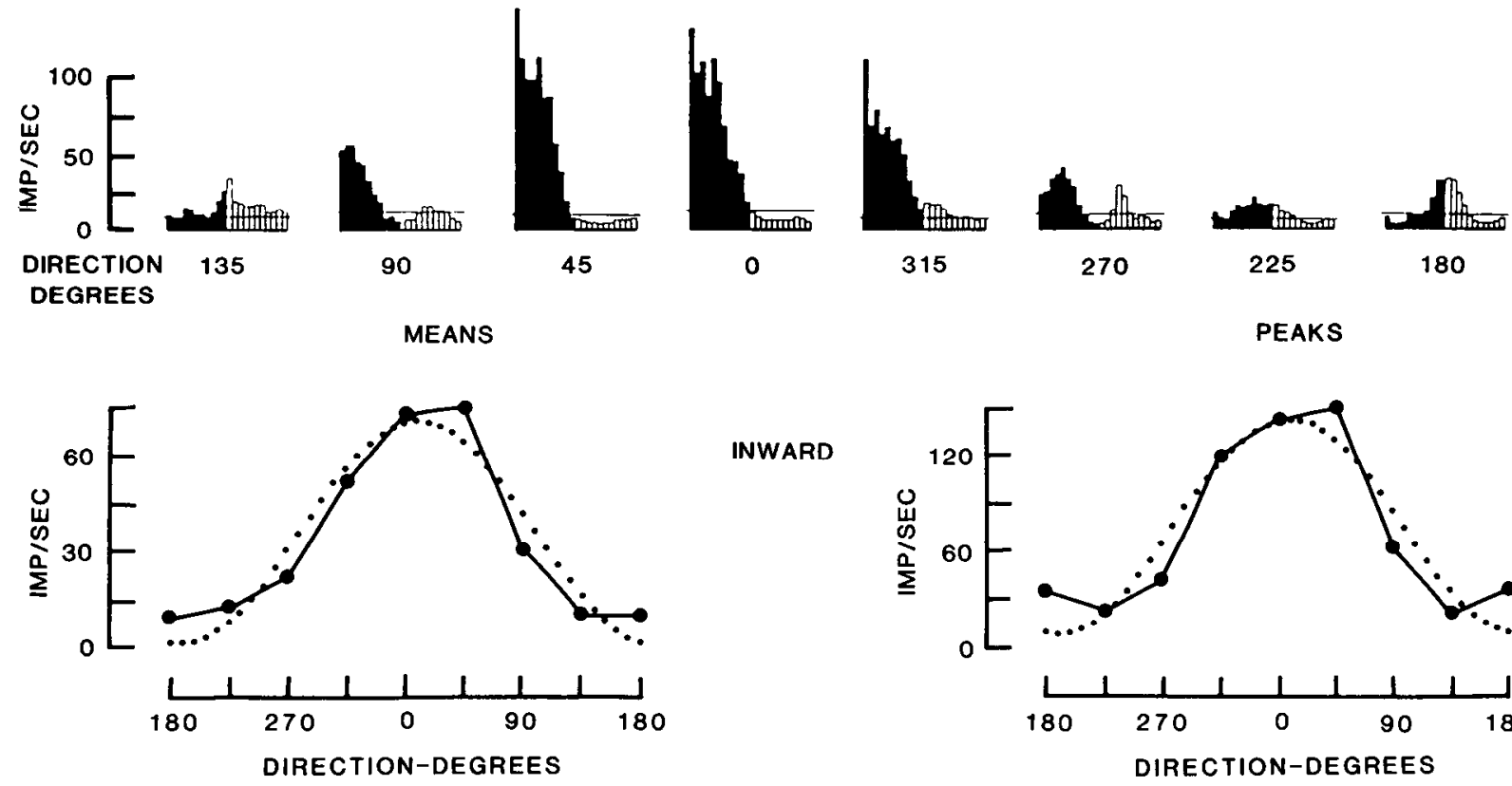

INWARD
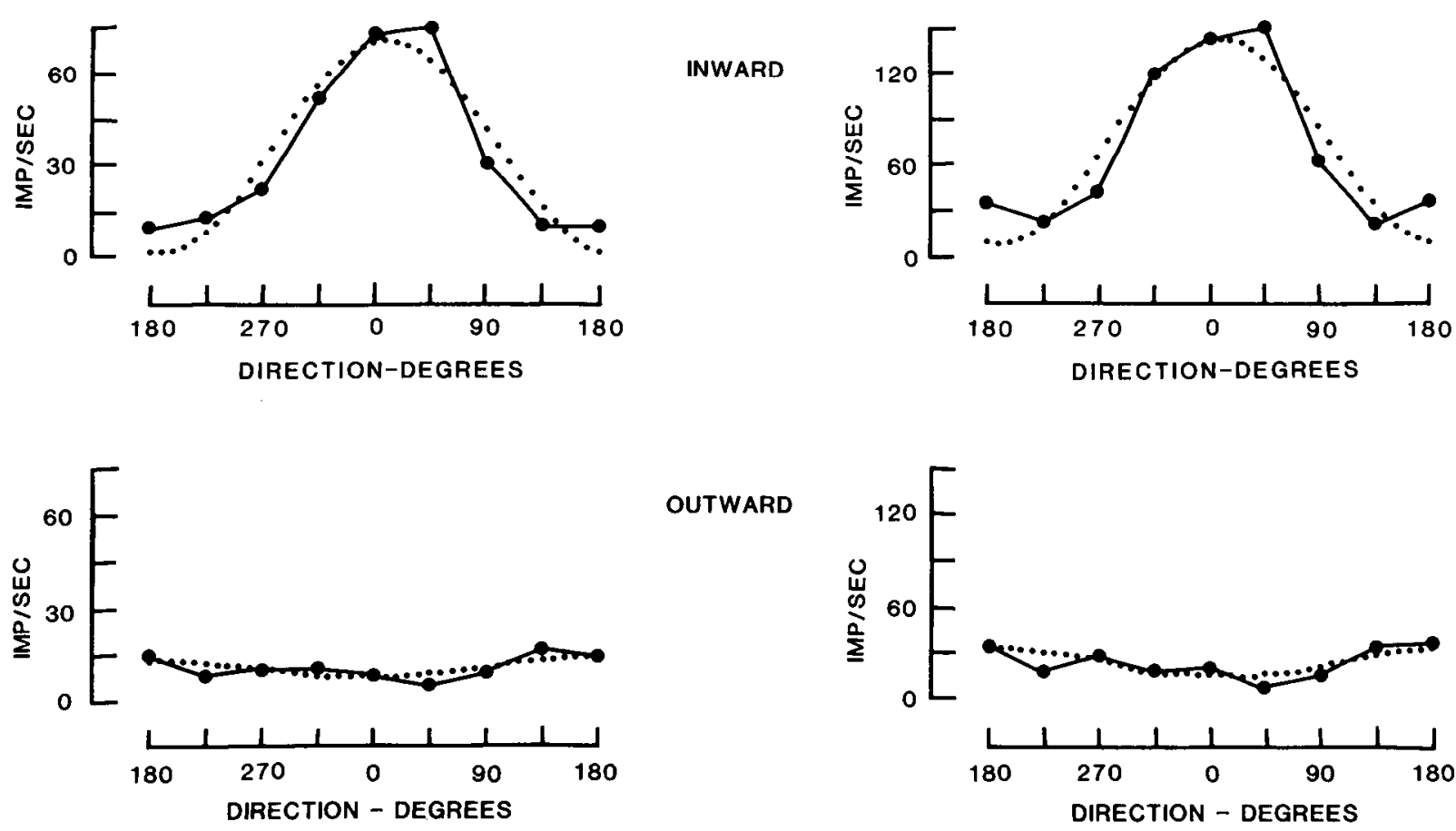

Figure 6. Sinusoidal variation in the amplitudes of responses of a PVN to moving stimuli. Histograms (top) show the time courses for the inward (dark shading) and outward (unshaded) halves of $100^{\circ}$ stimulus movements in each of the 8 directions indicated. The mean (left) and peak (right) responses are plotted as functions of the direction of stimulus movements for both the inward (middle) and outward (bottom) halves of stimulus movements. Solid lines connect data values; dotted lines show sine waves fitted to the data by periodic regression (see Materials and Mcthods).

tributions of their responses to radially moving stimuli. The preferred axes are given as the angle counterclockwise from the downward direction along the vertical meridian. Observations on cells from left hemispheres are reversed for the purposes of this figure. Although all directions of motion appear to be represented in the population from each hemisphere, there is a greater number of PVNs with best directions either toward or away from the fixation point into or out of the contralateral half of the visual field.

An index of angular tuning was calculated for all PVNs whose preferred directions are shown in Figure 10, using the $50 \%$ criterion described in Materials and Methods. The histogram of Figure 11 shows the distribution of these indices for the PVN population we analyzed. The median value is $90^{\circ}$, which corresponds to a $50 \%$ reduction in the amplitude of response for directions of motion $45^{\circ}$ to either side of the optimal direction. Eight cells of our population responded to stimuli moving along only a single axis and thus had tuning indices of $0^{\circ}$, while 27 cells had tuning indices $>180^{\circ}$. These data suggest that the signal of direction of stimulus motion is rather imprecise for individual PVNs and that if a more precise signal exists in the parietal lobe, it may be encoded in the population discharge.
In summary, the majority of parietal visual neurons with bilateral receptive fields show an opponent organization of directional preferences along meridians. For many, this preference remains either inward toward or outward away from the point of fixation for all meridians; i.e., the true angle of the directional preference changes from one meridian to another. The intensity of the responses does vary and is adequately described by a sinusoidal function fitted to the circle. These data suggest that the signal for the direction of stimulus motion is rather imprecise for individual PVNs; a more precise signal may be encoded in the PVN population discharge.

\section{Vectoral analysis of population directionality}

The proposition that a more precise signal of stimulus direction may be encoded in the response of the population of PVNs raises the problem of whether or how information about the nature of a stimulus or a behavioral event can be extracted from a population of relatively imprecise neuronal signals. A vectorial model of neuronal responses was used by Georgopoulos et al. (1983) in an analysis of the relation of the activity of motor cortical neurons to the direction of aimed arm movements, a problem somewhat similar to our own. The basic assumption 


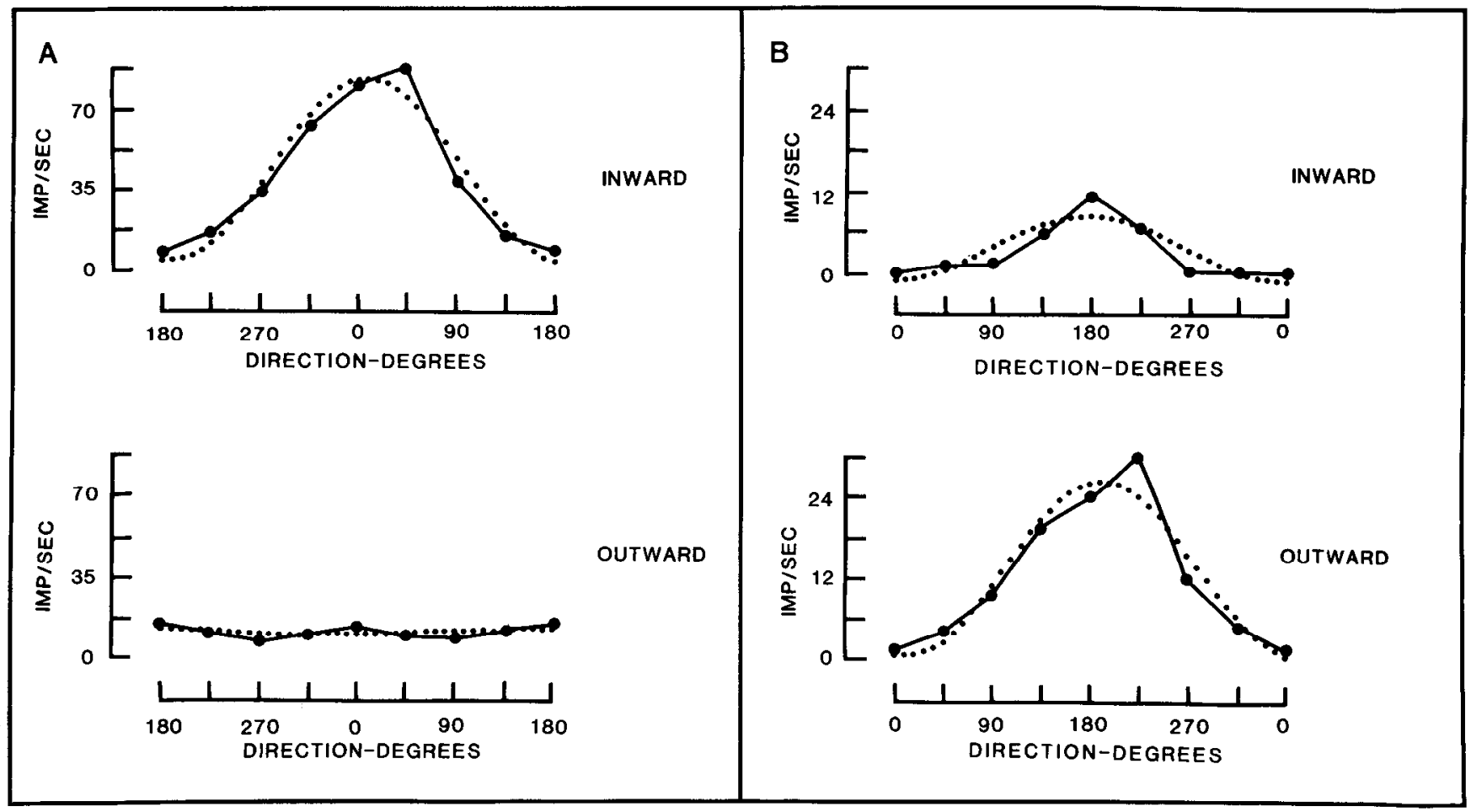

Figure 7. Sinusoidal regression plots of the responses of 2 PVNs, one of which $(A)$ responded preferentially to inward stimulus movements and the other of which $(B)$ responded preferentially to outward movements. See Figure 6 for details.

of the model is that a linear vectorial summation of directional responses of individual cortical neurons yields a population response that accurately predicts the direction of movement. We have applied this model to the responses of PVNs to visual stimuli moved either inward or outward along different radii symmetrically arranged around the central line of gaze in the frontoparallel plane normal to that line.

We calculated a single population vector for each direction of stimulus motion by the simple vector addition of the responses to all neurons in the population. The magnitude assigned to each neuronal vector was the difference between the rate of discharge as the stimulus moved in the test direction and

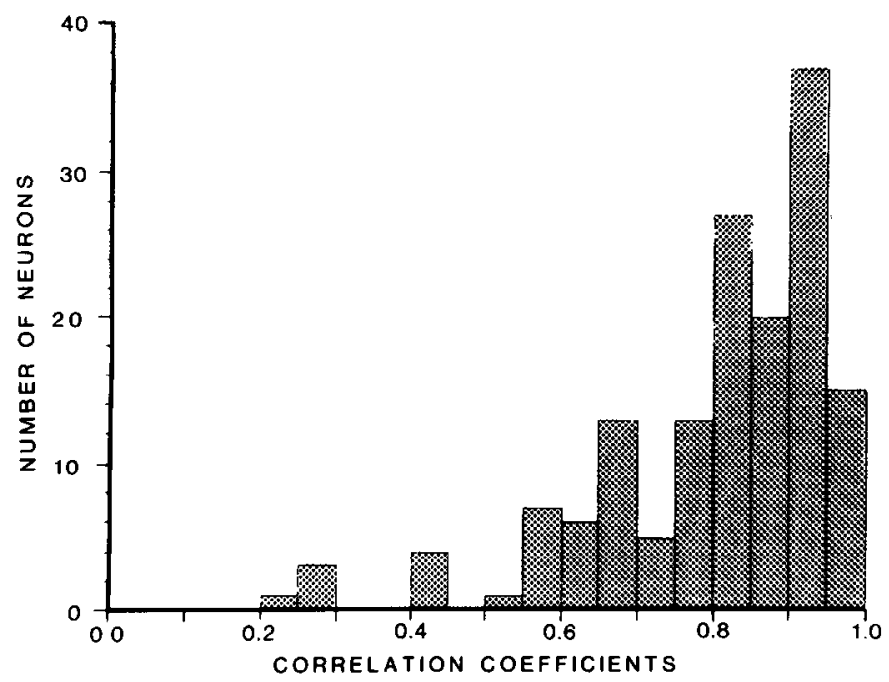

Figure 8. Distribution of correlation coefficients of sine waves fitted to data by periodic regression for the population of PVNs studied. the average response of that cell to all 8 directions of motion tested. The direction assigned to each neural vector was either its preferred direction, determined from sinusoidal regression analysis (see Materials and Methods), when responses were greater than the average rate, or the opposite direction, when responses were less than the average rate. Thus, each neuron contributed a vector in 1 direction along its preferred axis regardless of the direction of motion of the stimulus (the labeled line assumption) but with an amplitude that varied with direction of the test stimulus. The mean rates of discharge as the stimulus moved inward toward or outward away from the fixation target in the full-field tests (see Materials and Methods) were used for this analysis. A total of 104 of the 133 neurons that responded in these tests (see Table 1) were selective for a particular radial axis. Of that group, those with $r>0.7$ were analyzed in 4 sets: (1) PVNs that responded to inward stimulus motion only, (2) all PVNs that responded to inward motion along any meridian, (3) PVNs that responded to outward motion only, and (4) all PVNs that responded to outward motion along any meridian. The optimal directions for the sets of PVNs studied are not evenly distributed in the circular dimension for any 1 of the 4 groups, as shown by the inset diagrams of Figure 12 . This is most likely due to a sampling error owing to the relatively small number of neurons in each group. These nonuniform distributions of best directions, however, appear to have little or no effect on the capacity of the population to signal the direction of motion (Table 3, Fig. 13).

Individual and population vectors for the 8 directions of stimulus motion are shown separately for the 4 analysis groups in Figure 12. The directions of the resulting population vectors are indicated by arrows. The differences between the direction of the resultant population vectors and the actual directions of stimulus motion are summarized in Table 3. The graph of Figure 


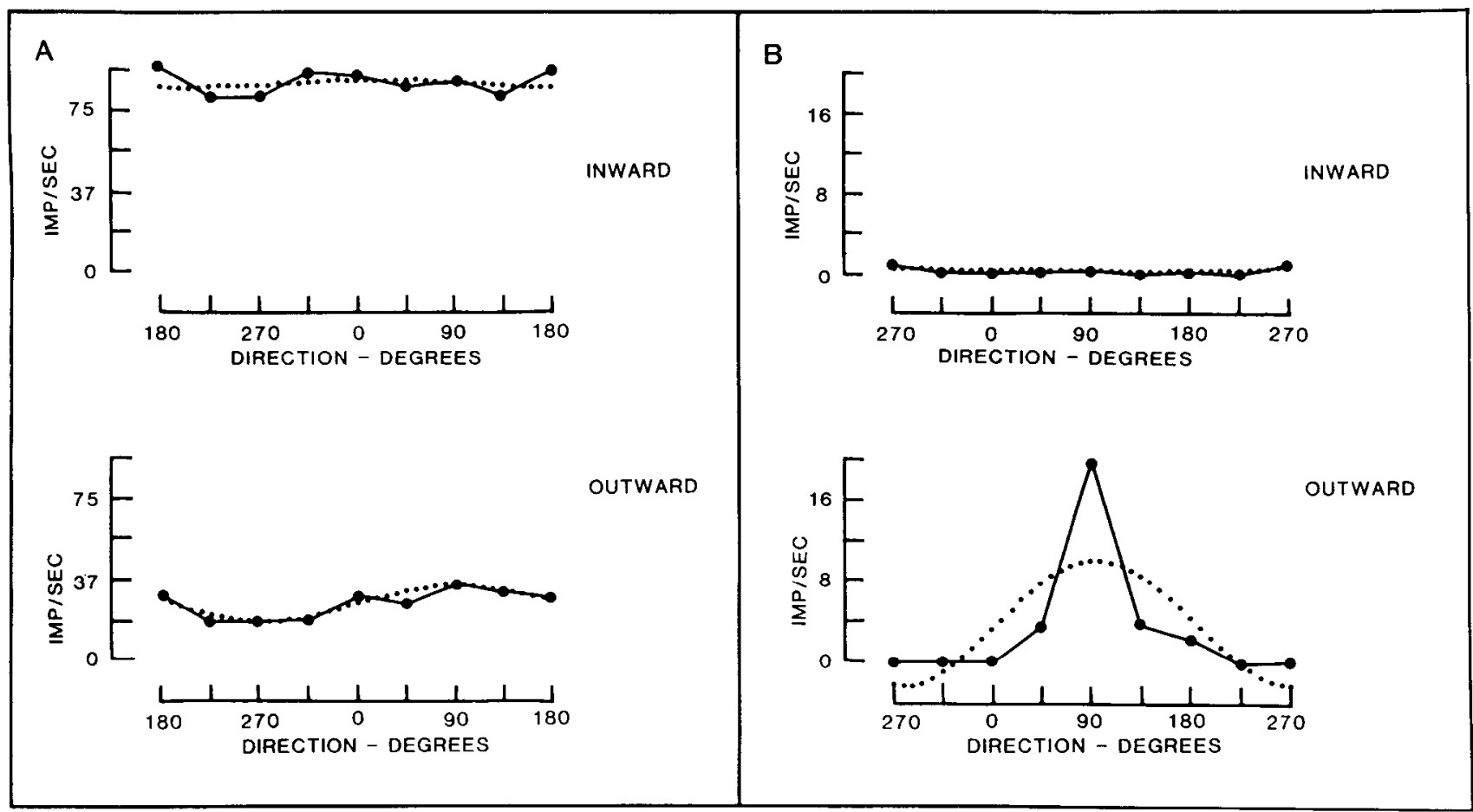

Figure 9. Sinusoidal regression for 2 parietal visual neurons for which the variation is not adequately described by a sinusoid $\left(r^{2}<0.70\right) . A$, Responses of a neuron similar to those of the neuron of Figure $2 A$, which are nearly equal for all inward directions of motion. $B$, Responses of a neuron "narrowly tuned" for outward motion.

13 shows that with this model the population response signals the direction of stimulus motion with great accuracy. In most cases, the direction of the population vector closely approximates the actual direction of stimulus motion; the average difference for the 4 groups is $9.3^{\circ}$. There is a closer correspondence between the directions of stimulus motion and the population vector for movements along the vertical and horizontal meridians (mean difference, $4.9^{\circ}$ ) than for those along diagonal axes (mean difference, $13.7^{\circ}$ ). Whether this is due to the uneven distribution of preferred directions in our neuronal sample or to real differences is unknown. The length of the population vector is a measure of the strength of the directional signal; it depends upon the number of contributing neurons and their discharge rates. Inspection of the values in any 1 group (Table 3) indicates some correlation between the resultant length, the symmetry in the underlying distribution (Fig. 12), and the difference in actual and predicted directions of stimulus motion. This suggests that the predictability of the model would be improved for a larger, more even sample of PVNs. Two other measures of dispersion, the mean resultant length and the circular standard deviation, can be calculated by the method of Mardia (1972); they are given in Table 3. These measures are proportional and normalized both for discharge rate and for the numbers of contributing elements, and thus are suitable for cross-comparison within and among groups. However, comparison of these measures of dispersion with the accuracy of prediction of stimulus direction by the population revealed no direct correlation.

\section{Discussion}

The observations described in this paper confirm the opponent organization of the directional preferences of PVNs. That is, individual PVNs respond on either the inward or the outward halves of stimulus movements along meridians of $100^{\circ}$ length, the latter centered on the point of fixation. The pattern of response is similar, either inward or outward for any given neuron, along all meridians tested. Analysis showed that the intensities

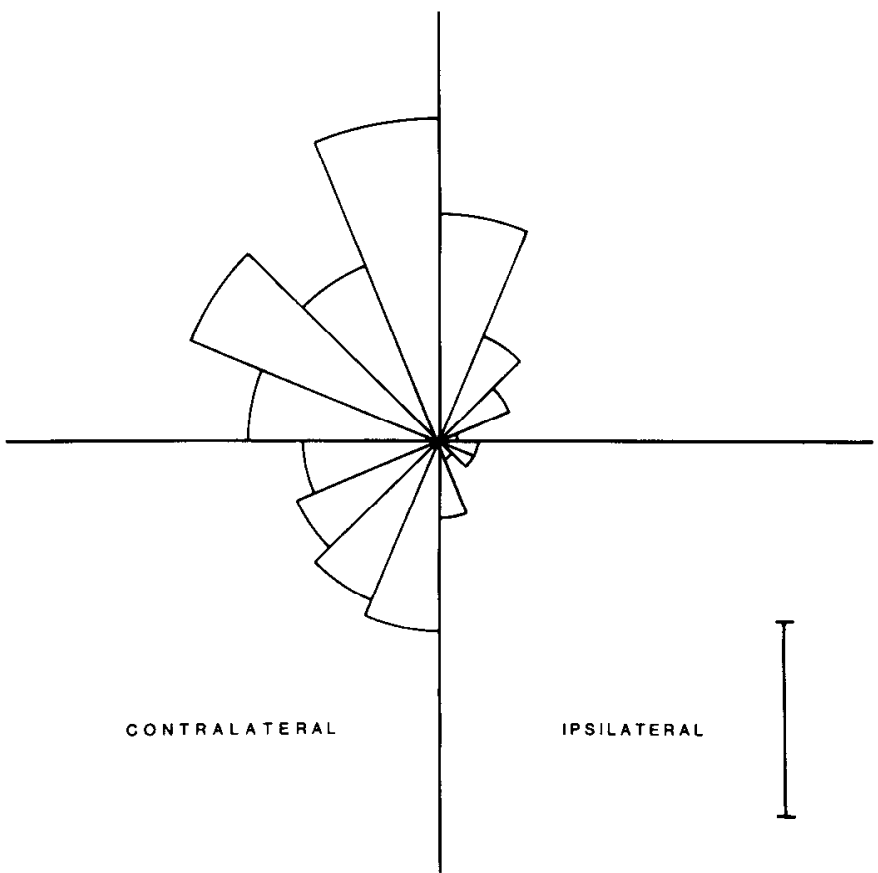

Figure 10. Rose diagram showing the distribution of the preferred directions (both inward and outward) in the population of PVNs studied. Observations on neurons in left hemispheres are reversed, producing this view of the population distribution seen from a right hemisphere. Vertical calibration bar indicates 10 cells. 


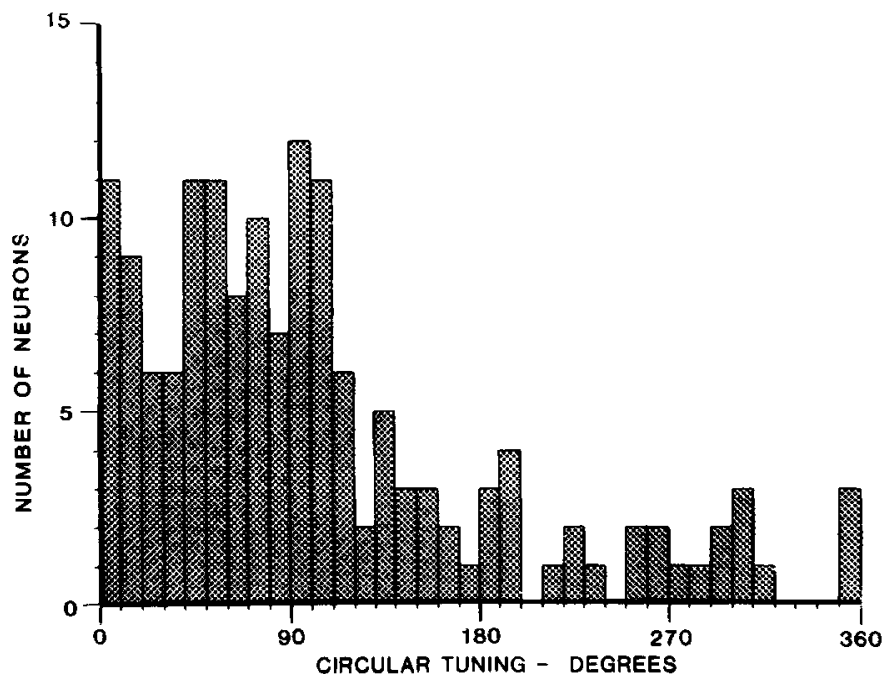

Figure 11. Distribution of the circular tuning indices in the population of PVNs studied. See Materials and Methods for description.

of response along those meridians varied systematically from 1 meridian to another, a variation described adequately by a sine wave function distributed on the circle. This suggests that, with rare exceptions, any single PVN is unlikely to provide a precise signal of the direction of a stimulus moving into or out of the visual field, toward or away from the central line of gaze. We therefore posed the question of whether a more precise signal of direction might be inherent in the population discharge of all neurons activated by such stimuli. An application of the vectorial model for the population signal of direction revealed that, with certain critical assumptions, this was indeed the case.

This discussion is based upon the experimental observations summarized above and upon the assumption that functions may be inferred from the physiological properties of neocortical cells observed in waking, behaving monkeys. This assumption is strengthened if the properties observed appear as positive images of the defects in function that occur after removal of the cortical area under study. We discuss first what mechanisms might produce the axial selectivity of PVNs for stimuli moving along meridians in a frontoparallel plane and propose a modification of the double-Gaussian model described in the preceding paper (Motter et al., 1987), which has, we believe, considerable explanatory value. We then discuss (1) the candidate neural code for stimulus direction embedded in the PVN population response, (2) the role of the parietal visual system in the visual guidance of projected movements of the arm and hand, and (3) the putative role of this system in the perception of selfmotion, in providing the signals used in guiding locomotion, and in evoking the illusion of vection.

Mechanism of axial selectivity in the circular dimension of the visual field

A visual stimulus moving in the null direction along a meridian evokes in PVNs a feed-forward inhibition that extends from the

Table 3. Results of the population vector analysis for each of the 8 directions of motion in the 4 groups shown in Figure 12

\begin{tabular}{|c|c|c|c|c|c|c|c|c|}
\hline \multirow{2}{*}{ Parameter } & \multicolumn{8}{|c|}{ Direction of motion } \\
\hline & $0^{\circ}$ & $45^{\circ}$ & $90^{\circ}$ & $135^{\circ}$ & $180^{\circ}$ & $225^{\circ}$ & $270^{\circ}$ & $315^{\circ}$ \\
\hline \multicolumn{9}{|c|}{ A. Neurons responding to inward motion only $(n=47)$} \\
\hline Population vector & 0 & 26 & 107 & 148 & 181 & 202 & 267 & 328 \\
\hline Difference & 0 & 19 & 17 & 13 & 1 & 23 & 33 & 13 \\
\hline Resultant length & 385 & 244 & 182 & 250 & 317 & 250 & 176 & 278 \\
\hline Mean resultant length & 0.84 & 0.71 & 0.63 & 0.73 & 0.82 & 0.76 & 0.62 & 0.76 \\
\hline Circular SD & 33 & 47 & 55 & 45 & 36 & 42 & 56 & 42 \\
\hline \multicolumn{9}{|c|}{ B. All neurons responding to inward stimulus motion $(n=78)$} \\
\hline Population vector & 358 & 31 & 91 & 144 & 184 & 215 & 274 & 326 \\
\hline Difference & 2 & 14 & 1 & 9 & 4 & 10 & 4 & 11 \\
\hline Resultant length & 505 & 372 & 346 & 407 & 473 & 428 & 264 & 380 \\
\hline Mean resultant length & 0.81 & 0.74 & 0.65 & 0.72 & 0.79 & 0.76 & 0.60 & 0.71 \\
\hline Circular SD & 37 & 44 & 53 & 47 & 39 & 42 & 58 & 47 \\
\hline \multicolumn{9}{|c|}{ C. Neurons responding to outward stimulus motion only $(n=12)$} \\
\hline Population vector & 344 & 49 & 93 & 140 & 202 & 226 & 265 & 301 \\
\hline Difference & 16 & 4 & 3 & 5 & 18 & 1 & 5 & 14 \\
\hline Resultant length & 44 & 55 & 85 & 36 & 36 & 47 & 52 & 46 \\
\hline Mean resultant length & 0.69 & 0.78 & 0.79 & 0.64 & 0.62 & 0.70 & 0.75 & 0.69 \\
\hline Circular SD & 50 & 40 & 39 & 54 & 56 & 49 & 43 & 50 \\
\hline \multicolumn{9}{|c|}{ D. All neurons responding to outward stimulus motion $(n=42)$} \\
\hline Population vector & 4 & 28 & 90 & 163 & 181 & 200 & 270 & 328 \\
\hline Difference & 4 & 17 & 0 & 28 & 1 & 25 & 0 & 13 \\
\hline Resultant length & 285 & 211 & 126 & 197 & 215 & 226 & 130 & 169 \\
\hline Mean resultant length & 0.85 & 0.78 & 0.58 & 0.77 & 0.82 & 0.80 & 0.54 & 0.69 \\
\hline Circular SD & 33 & 40 & 60 & 41 & 36 & 38 & 63 & 49 \\
\hline
\end{tabular}

The resultant length, the mean resultant length, and the circular SD are measures of dispersion (see text). 


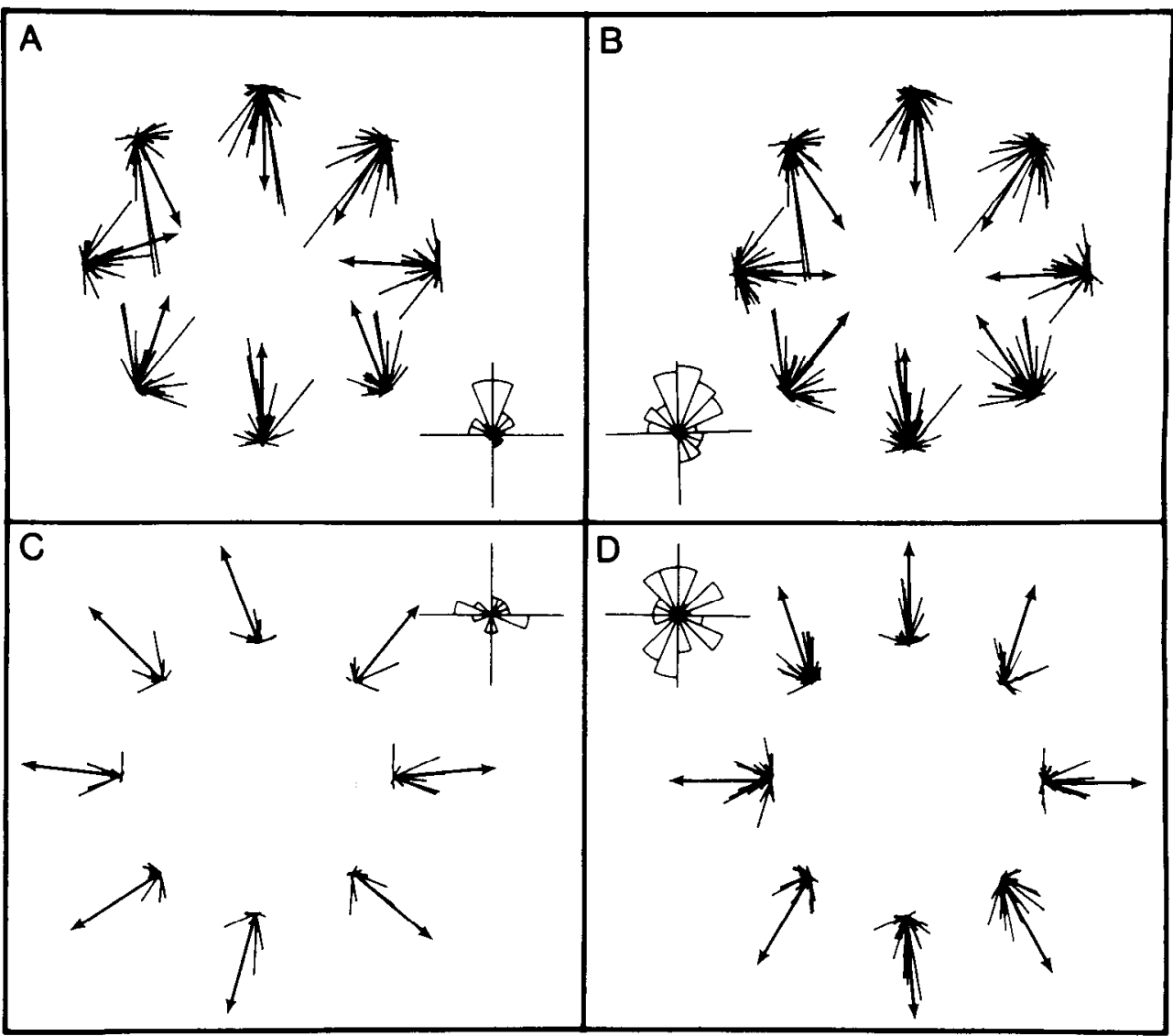

Figure 12. Individual response vectors and the resulting population vectors (arrows) for the population of PVNs studied for each of the 8 directions of stimulus motion. $A$, Cells responding to inward motion only. $B$, All cells responding to inward motion. $C$, Cells responding to outward motion only. $D$, All cells responding to outward motion. Insets show the distributions of the preferred directions for each set analyzed. Length of maximum radius in insets $A-$ $D$ represents 12,12 , and 6 neurons, respectively. Detailed description of the analysis is given in the text. leading edge of the stimulus forward for $20^{\circ}-30^{\circ}$. This inhibition is assumed to be generated by intracortical circuit action. A model was presented (Fig. 16, Motter et al., 1986) that accounts for the opponent directionalities observed when stimuli move in the 2 directions along meridians of $100^{\circ}$ extent, with gaze fixed at the center. Each PVN is assumed to be related to large inhibitory and excitatory receptive fields that are symmetrically superimposed and Gaussian in distribution but of unequal size. When the excitatory field is the larger, the inward pattern of directional preference results. The initial stimulus movement inward from the periphery evokes strong excitation and, with a delay, slowly summating and persisting inhibition that quenches the response as the stimulus reaches (approaches) the central line of gaze, and prevents response on the outward half of stimulus movement, away from the point of fixation. Reciprocal interpretations account for the directional properties of PVNs responding to outward stimulus motion. Several lines of experimental evidence were presented that support the validity of this model.

This same model accounts for the fact that the true directions of the directional preferences along the meridians change sharply with changes in the angle of the meridians tested for the majority of PVNs. The overlapping but unequal spatial extents of the excitatory and inhibitory fields account for the facts observed, for the results will be the same whatever the meridional angle of stimulus entry from the periphery. The observation that no consistent differences in local directional preferences exist in different parts of the receptive fields lends complementary support to the explanation offered.

However, we observed only 3 neurons (see Figs. $2 A$ and $9 A$ ) whose receptive fields and response intensities were sufficiently symmetric in the circular dimension of the frontoparallel plane to fit the model precisely. For the majority of PVNs the rule of inward or outward directional preference held along all meridians, but the intensities of the responses and the spatial extent of the receptive field differed for different meridians. We propose a modification of our original model to account for these asym-

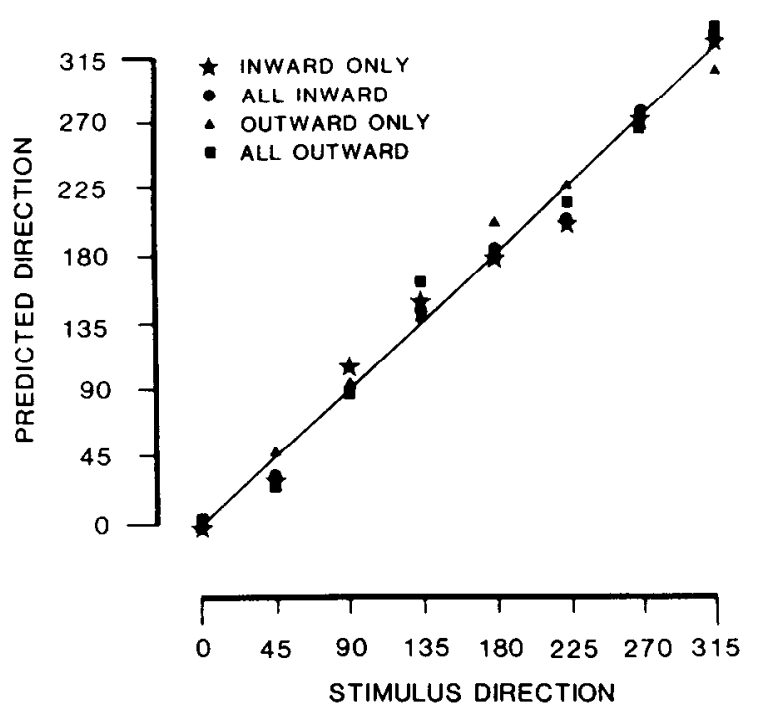

Figure 13. Population vectors (predicted directions) shown as functions of the actual stimulus directions for each of the $\mathbf{4}$ groups of Figure 12. 
Figure 14. Graphic representation of the responses of a PVN to inwardly moving stimuli. This response surface is the sum of 2 Gaussian functions, 1 excitatory and 1 inhibitory. The asymmetry results from slightly shifting the peak of the inhibitory function relative to that of the excitatory one.

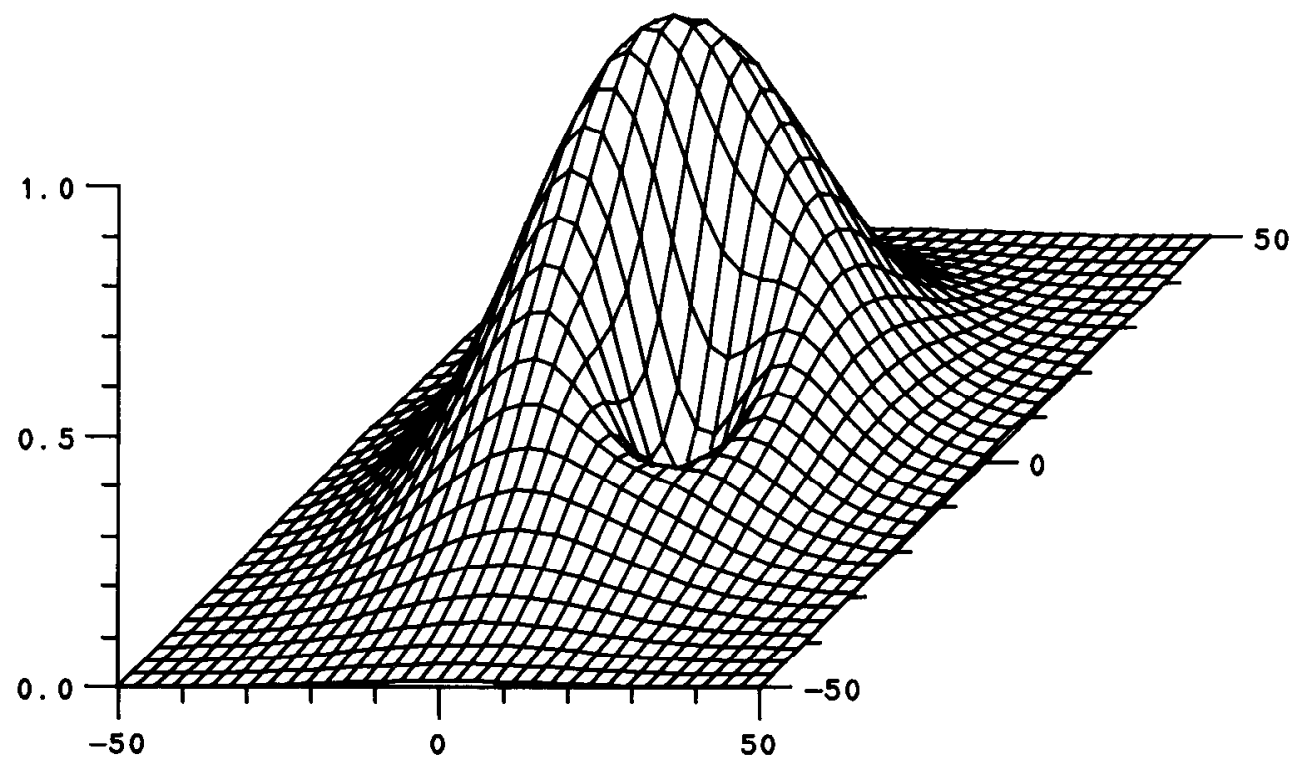

metries; it is shown in Figure 14, where the original symmetric distribution of the Gaussians is distorted to account for the asymmetric distribution of response intensities observed. An analysis of responses to motion along all the meridians tested revealed that they are accounted for with a high level of confidence by a sinusoidal function fitted in the circular dimension (Fig. 8). This allowed us to calculate from the sinusoidal distribution the best or preferred meridional direction of stimulus motion for each PVN. PVNs are "broadly tuned" for direction, such that responses significantly greater than the average for all stimulus directions occur up to $45^{\circ}$ on either side of the best direction (Fig. 11). We therefore sought to determine by analysis whether a more precise signal of the meridional direction of stimulus motion is coded in the pattern of discharge of the full population of PVNs activated by a given stimulus.

A code for stimulus direction embedded in the PVN population pattern of response

A general problem in neurophysiology is whether precise signals of events may be coded in population patterns of neuronal activity, when only imprecise signals of those events are provided by any single neuron of the population. Some mechanism of this sort appears necessary to account for the common observation that sensory and motor performances are more exact than would be predicted from the signal imprecision of single neurons. The broad distribution of responses to stimuli moving along different meridians (see Fig. 11) suggests that this may be the case for the PVN population. We have tested this proposition by an application of the population vector summation model. The main assumption of the model in the present application is that the discharge of any single PVN, regardless of the direction of the stimulus activating it in any given case, provides a signal of stimulus movement along the preferred axis of the cell, in the preferred direction if response magnitude is above, and in the opposite direction if below, the average response to all directions of motion tested. We refer to this as the labeled line assumption. We then carried out a linear vector summation of the responses of all neurons in the population considered for each direction of stimulus motion tested.

The results obtained are illustrated in Figures 12 and 13 and detailed in Table 3. The derived population vector responses differed on average by only $9.3^{\circ}$ from the actual direction of stimulus motion. This precise signal contrasts with the broad and relatively imprecise signal of the direction stimulus movement provided by any single PVN of the population analyzed. It caused us to examine further the assumptions involved in the application of the model.

The first is the labeled line assumption described above. It appears to us to be inherently as likely as the ubiquitous modality specificity demonstrated repeatedly in the sensory systems of mammalian brains. The second assumption is that there exist neuronal mechanisms for recognizing labeled lines and for linear vector summation. No such mechanisms have, to our knowledge, been described, but their existence appears to us more plausible than the alternative, i.e., that summations of this kind are made by single neurons or even by small groups of neurons. It seems more likely that the flow-through of brain activity from inputs to outputs occurs by the interfacing of large neuronal populations, although little is presently known of the nature of those interfaces.

The choice of the average response to all directions of stimulus motion as the reference level and the assignment of all signals above that level to the preferred axis and direction of the cell, and all those below that level to the preferred axis but in the opposite direction along that same preferred axis, assure the good fit of the model to our data. However, we believe the use of the average as reference level would not be necessary under certain conditions. If, for example, the spontaneous rate of discharge of each PVN in the absence of visual stimulation were used as the basis for measurement, the results of the analysis would be expected to be quite similar, if (1) there existed a uniform distribution of neuronal best directions around the circle, (2) the directional variance of the responses of PVNs with different best directions were similar, and (3) the peak discharge rates of different PVNs were similar. It is likely these conditions would be met with a larger experimental sample. Thus, we believe the result we obtained does not depend critically upon the choice of reference level.

We conclude that there is a more precise signal of the meridional direction of stimulus movement embedded in the population 
discharge of PVNs than exists in the discharge of any single member of the population. The vector summation model and analysis provide an exact description of this population signal, but whether the signal expressed as a vector can be detected and used by other neuronal populations remains to be established.

\section{Role of the parietal visual system in the perception of self-motion and in the visual guidance of locomotion}

Linear motion of the head or rotation of the eye within the head results in a predictable motion of a static visual scene known as the "optic flow field" (Gibson, 1966). Information derived from this optic flow is thought to be important for the perception of self-motion, for distinguishing self-motion from object motion, and for the visual guidance of locomotion.

The perception of self-motion by humans is produced largely by movement of the object surround through the periphery of the visual field. Motion in the visual periphery elicits in a stationary observer an illusion of self-motion (vection) indistinguishable from real motion. The illusion survives blocking of the central $60^{\circ}$ of the visual field but is degraded by masking peripheral vision (Brandt et al., 1973; Johansson, 1977; McKee and Nakayama, 1984). The illusion of vection is compelling, for it dominates contradictory proprioceptive signals. For example, subjects presented with optical flow consistent with backward self-motion perceive backward motion even if they are actually walking forward (Lishman and Lee, 1973; Lee and Thomsen, 1982). Similarly, under some conditions optic flow signals are crucial for the control of stationary balance (Lee and Lishman, 1975; Amblard and Carblanc, 1980).

Humans can direct their gaze to within $30 \mathrm{~min}$ of arc of their direction of locomotion (Priest and Cutting, 1985). When only linear translation occurs, the direction of locomotion could be determined by directing the angle of gaze such that the lateral optic flows are symmetrical on the 2 sides; the task is more difficult if translation is combined with rotation. The human capacity to detect the focus of expansion of the optic flow field is limited, so that it is unlikely to serve effectively to guide the direction of locomotion (Regan and Beverly, 1982). It appears that the instantaneous velocity field in the visual periphery is used by humans in tasks that require critical judgment of the direction of movement, such as the guidance of high-speed land vehicles or the landing of aircraft (Gordon and Michaels, 1963; Gordon, 1965; Regan and Beverly, 1982).

These optic flow patterns will evoke maximal rates of discharge of PVNs because of the wide, bilateral receptive fields of these neurons and their history-dependent opponent directionality observed when stimuli traverse meridians that pass through the fixation point (Motter and Mountcastle, 1981; Mountcastle et al., 1984; Motter et al., 1987). Moreover, steady fixation of the point ahead induces a powerful facilitation of the responses of PVNs to visual stimuli moving inward or outward along axis meridians (Mountcastle et al., 1981). Linear motion through a fixed environment will evoke maximal, balanced levels of activity in the PVN populations of both hemispheres. Forward and backward self-motion will be signaled differentially by the inward and outward motion-sensitive subsets of PVNs when gaze is directed along the line of movement, especially when a distant target is fixated. Under either circumstance, the optic flow array will contain stimuli moving along all meridians; thus, each PVN will be driven by its preferred stimulus direction. The excitatory drive will derive from all stimuli in the surround, from those moving slowly near the center of gaze to those moving rapidly at the periphery of the visual fields. This is so because PVNs, while exquisitely sensitive to stimulus motion and direction, are relatively insensitive to differences in stimulus speed and respond over a wide range of speeds (Motter et al., 1987). We have no evidence for hemispheric differences in the distribution of preferred directions of PVNs in the monkey. However, the contralaterality of the visuospatial defects in humans with parietal lobe lesions suggests that such a differential distribution may occur in humans. If so, the bilateral symmetry of activity in the PVN population during linear motion in the line of gaze will be further enhanced.

If the direction of motion is at an angle to the line of gaze, the PVN population signal of the apparent motion of the surround will be quite different. For example, if locomotion is at some angle to the right of the line of gaze, PVNs with best directions pointed outward in the left visual field will be driven as described above. Neurons sensitive to outwardly moving stimuli, with best directions in the right half of the visual field lateral to the line of locomotion, will be excited, as will inwardly sensitive cells with best directions between the line of gaze and the line of locomotion. This shearing motion will evoke a powerful differential activation of the groups of PVNs sensitive to outward and inward stimulus movement. However, the effects of this simultaneous outward and inward stimulus motion in the same half of the visual field cannot be predicted from the facts presently available. Our preliminary studies of PVNs when 2 moving stimuli are presented simultaneously in the visual field indicate that the effect will be determined by nonlinear interactions (V.B. Mountcastle, B.C. Motter, and M.A. Steinmetz, unpublished observations). Nor can the influence of the deviation of the angle of gaze upon the overall PVN population response be assessed at present. Although powerful facilitatory and suppressive effects of deviation of the eyes upon the excitability of PVNs occur (Andersen and Mountcastle, 1983), a systematic description of these effects in terms of laterality is not yet available.

The relative insensitivity of PVNs to stimulus speed ensures a continuous excitatory drive independent of the range of velocities of the apparent optic flow during self-motion. However, we have not studied the sensitivity of PVNs to accelerating stimuli nor to differential rates or directions of multiple stimuli such as those that provide kineoptic depth signals (Nakayama and Loomis, 1974; Frost and Nakayama, 1983). It has recently been shown, however, that many motion-sensitive neurons of area MT, which projects indirectly upon area PG, are differentially activated or suppressed depending upon the "against" or "with" direction of motion of the background vs that of the stimuli (Allman et al., 1985; Saito et al., 1986; Tanaka et al., 1986), an effect thought important for the discrimination of selffrom object motion. We have not examined PVNs under those stimulus conditions.

\section{Role of the parietal visual system in the visual guidance of projected movements of the arm and hand}

It has been known since the time of Balint (1909) that lesions of the posterior parietal cortex in man produce defects in the accuracy of reaching movements of arm and hand to targets; a similar defect is causcd by lesions of the postcrior parictal cortex in nonhuman primates (for reviews, see Humphrey, 1979; Hyvarinen, 1982; Andersen, 1987; Georgopoulos, 1986). This optic ataxia is distinguished from the visual disorientation of 
Holmes (1918) that may follow somewhat similarly located lesions in man (Perinen and Vighetto, 1983). The projection by primates of the arm and hand towards a target is a smooth combination of 2 movements. The first is a rapid, semiballistic projection of the arm, the second an adaptive shaping of the hand to the target during and at the end of the arm projection (Jeannerod and Biguer, 1982). Both components are defective after parietal lobe lesions in man. Normal projected movements occur along paths with segments of different curvature; the paths are unaffected by constant loads of different value or by variations in movement specd (Soechting and Lacquaniti, 1981). The velocity profile of movement is dome-shaped, with a peak at about $100 \mathrm{~cm} / \mathrm{sec}$ (Beggs and Horwarth, 1972).

Psychophysical studies of visual motion perception in man indicate the existence of 2 superimposed visual motion detecting systems. One derives motion from successive positions and is distributed throughout the visual field but is most potent in the foveal region. The second system is directly sensitive to moving targets and is distributed throughout the visual field but is most potent in the periphery. While visual sensitivity to pattern and to discrete motion declines with eccentricity, that to continuous motion is invariant with eccentricity (Bonnet, 1977, 1981). Several experimental observations support the general thesis of Paillard that these 2 visual motion detection systems provide the afferent limbs for the visual guidance and control of the 2 components of projected movements of the arm and hand: the peripherally dominant "movingness" system for the initial, semiballistic movement of the arm; the centrally dominant displacement detection system for the adaptive shaping of the hand to the target (Conti and Beaubaton, 1976; Paillard and Beaubaton, 1976; Beaubaton et al., 1977; Prablanc et al., 1979a, b; Paillard, 1980, 1982; Paillard and Amblard, 1984). Adaptation of the projected movement of the arm to a prismatically displaced target is greatly delayed in stroboscopic lighting. Cues evoked by passive movement of the arm are not processed, which suggests that it is the engagement of the central neural components of the control loop in afferent-efferent linkage that is important for the visual guidance of the movement (Paillard et al., 1981).

The role of the parietal system in the visual guidance of projected arm movements depends in the first instance, we propose, on the afferent input provided by PVNs, a function to which they appear well suited by virtue of their special properties. The inward directionality of the majority $(75 \%)$ of PVNs provides a large population of neurons tuned to signal the movement of the arm from the periphery of the visual field towards a centrally fixated target. The wide range of speed sensitivity of PVNs is matched to the dome-shaped profile of velocities of projected movements. PVNs will thus provide continuous signals as the velocity of the arm increases and decreases. The large size of PVN receptive fields and the insensitivity of PVNs to detailed features of moving stimuli mean that PVNs will respond smoothly and continuously throughout the projected movement. The population vector described above provides a precise signal of the direction of the movement of the arm through the visual field. Finally, fixation of a central target facilitates the responses of PVNs to stimuli moving through the periphery of the visual field (Mountcastle et al., 1981).

The posterior parietal cortex of monkeys contains, in areas 5 and $7 \mathrm{a}$, large populations of neurons that discharge before and during projected movements of the arm and less intensely or indeed not at all to other movements of the arm (Mountcastle et al., 1975). A fraction of these cells continue to discharge with projected movements of denervated limbs, which suggests the subset is activated via centrally reentrant circuits (Bioulac and LaMarre, 1979; Seal et al., 1982). Another subset of the projection neurons is also sensitive to sensory cueing stimuli (Seal and Commenges, 1985), which suggests that PVNs and the projection neurons may form the central end of the error-detecting and control loop that regulates the direction of projected movements of the arm and hand.

A class of PVNs with smaller, foveally centered receptive fields has been identified but not studied in detail (Motter and Mountcastle, 1981). They respond intensely to stationary stimuli. It is reasonable to suggest that this particular set of PVNs combines with the manipulation neurons of the parietal cortex to form the central components of the neural loops regulating the adaptive shaping of the hand to a target at the end of projected movements.

\section{References}

Allman, J., F. Miezin, and E. McGuiness (1985) Stimulus specific responses from beyond the classical receptive field: Neurophysiological mechanisms for local-global comparisons in visual neurons. Annu. Rev. Neurosci. 8: 407-430.

Amblard, B., and A. Carblanc (1980) Role of foveal and peripheral vision information in the maintenance of postural equilibrium in man. Percept. Mot. Skills 51: 903-912.

Andersen, R. A. (1987) The Role of the Inferior Parietal Lobule in Spatial Perception and Visual Motion Integration, American Physiological Society (in press).

Andersen, R. A., and V. B. Mountcastle (1983) The influence of the angle of gaze upon the excitability of the light-sensitive neurons of the posterior parietal cortex. J. Neurosci. 3: 532-548.

Balint, R. (1909) Seelenlahmung des "Schauens," optische Ataxie raumliche Storung der Aufmerksamkeit. Monatschr. Psychiatr. Neurol. 25: 51-81.

Batschelet, E. (1981) Circular Statistics in Biology, Academic, New York.

Beaubaton, D., A. Grangetto, and J. Paillard (1977) Contribution of positional and movement cues to visuo-motor reaching in split-brain monkey. In Structure and Function of Cerebral Commissures, I. StelleRussel, M. W. van Hoff, and G. Berluchi, eds., pp. 371-384, MacMillan, London.

Beggs, W. D. A., and C. I. Horwarth (1972) The movement of the hand towards a target. Q. J. Exp. Psychol. 24: 448-453.

Bioulac, B., and Y. LaMarre (1979) Activity of postcentral cortical neurons of the monkey during conditioned movements of a deafferented limb. Brain Res. 172: 427-437.

Bonnet, C. (1977) Visual motion detection models: Features and frequency filters. Perception 6: 491-500.

Bonnet, C. (1981) Thresholds of motion perception. In Tutorial in Motion Perception, A. H. Wertheim, A. A. Wagenaar, and H. W. Leibowitz, eds., pp. 41-79, Plenum, New York.

Brandt, T., J. Ditchgans, and E. Koenig (1973) Differential effects of central versus peripheral vision on egocentric and exocentric motion perception. Exp. Brain Res. 16: 476-491.

Conti, P., and D. Beaubaton (1976) Utilisation des informations visuelles dans le contrôle du mouvement: Etude de la précision des pointages chez l'homme. Travail Humain 39: 19-32.

Frost, B. J., and K. Nakayama (1983) Single visual neurons codc opposing motion independent of direction. Science 220: 744-745.

Georgopoulos, A. P. (1986) On reaching. Annu. Rev. Neurosci. 9: 147-170.

Georgopoulos, A. P., R. Caminiti, J. F. Kalaska, and J. T. Massey (1983) Spatial coding of movement: A hypothesis concerning the coding of movement direction by motor cortical populations. Exp. Brain Res. [Suppl.] 7: 327-336.

Gibson, J. J. (1966) The Senses Considered as Perceptual Systems, Houghton-Mifflin, Boston.

Gordon, D. A. (1965) Static and dynamic visual fields in human space perception. J. Opt. Soc. Am. 55: 1296-1303.

Gordon, D. A., and R. M. Michaels (1963) Static and dynamic visual 
fields in vehicular guidance. Highway Res. Rec. 84: 1-15.

Holmes, G. (1918) Disturbances of visual orientation. Br. J. Ophthalmol. 2: 449-468, 506-516.

Humphrey, D. R. (1979) On the cortical control of visually directed reaching: Contributions by non-precentral motor areas. In Posture and Movement, R. E. Talbott and D. R. Humphrey, eds., pp. 51112, Raven, New York.

Hyvarinen, J. (1982) The Parietal Cortex of Monkey and Man, Springer, New York.

Jeannerod, M., and B. Biguer (1982) Visuomotor mechanisms in reaching within extrapersonal space. In Analysis of Visual Behavior, D. J. Ingle, M. A. Goodale, and R. J. W. Mansfield, eds., pp. 387409, MIT Press, Cambridge, MA.

Johansson, G. (1977) Studies on visual perception of locomotion. Perception 6: 365-376.

Lee, D. N., and J. R. Lishman (1975) Visual proprioceptive control of stance. J. Hum. Movement Stud. 1: 87-95.

Lee, D. N., and J. A. Thomsen (1982) Vision in action: The control of locomotion. In Analysis of Visual Behavior, D. J. Ingle, M. A. Goodale, and R. J. W. Mansfield, eds., pp. 411-433, MIT Press, Cambridge, MA.

Lishman, J. R., and D. N. Lee (1973) The autonomy of visual kinaesthesis. Perception 2: 287-294.

McKee, S. P., and K. Nakayama (1984) The detection of motion in the peripheral visual field. Vision Res. 24: 25-32.

Mardia, K. V. (1972) Statistics of Directional Data, Academic, New York.

Motter, B. C., and V. B. Mountcastle (1981) The functional properties of the light-sensitive neurons of the posterior parietal cortex studied in waking monkeys: Foveal sparing and opponent vector organization. J. Neurosci. 1: 3-26.

Motter, B. C., M. A. Steinmetz, C. J. Duffy, and V. B. Mountcastle (1987) Functional properties of parietal visual neurons: Mechanisms of directionality along a single axis. J. Neurosci. 7: 154-176.

Mountcastle, V. B., J. C. Lynch, A. Georgopoulos, H. Sakata, and C. Acuna (1975) Posterior parietal association cortex of the monkey: Command functions for operations within extra-personal space. J Neurophysiol. 38: 871-908.

Mountcastle, V. B., R. A. Andersen, and B. C. Motter (1981) The influence of attentive fixation upon the excitability of the light-sensitive neurons of the posterior parietal cortex. J. Neurosci. 1: 12181235.

Mountcastle, V. B., B. C. Motter, M. A. Steinmetz, and C. J. Duffy (1984) Looking and seeing: The visual functions of the parietal lobe. In Dynamic Aspects of Neocortical Function, G. M. Edelman, W. E. Gall, and W. M. Cowan, eds., pp. 159-194, Wiley, New York.

Nakayama, K., and J. M. Loomis (1974) Optical velocity patterns, velocity-sensitive neurons, and space perception: A hypothesis. Perception 3: 63-80.

Paillard, J. (1980) Multichannelling of visual cues and the organization of visually guided responses. In Tutorials in Motor Behavior, G. E.
Stelmach and J. Requin, eds., pp. 259-279, North-Holland, Amsterdam.

Paillard, J. (1982) The contribution of peripheral and central vision to visually guided reaching. In Analysis of Visual Behavior, D. J. Ingle, M. A. Goodale, and R. J. W. Mansfield, eds., pp. 367-385, MIT Press, Cambridge, MA.

Paillard, J., and B. Amblard (1984) Static versus kinetic visual cues in the processing of spatial relationships. In Brain Mechanisms of Spatial Vision, D. J. Ingle, M. Jeannerod, and D. N. Lee, eds., pp. 299-330, Martinus Nijhof, The Hague.

Paillard, J., and D. Beaubaton (1976) Triggered and guided components of visual reaching. Their dissociation in split-brain studies. In The Motor System: Neurophysiology and Muscle Mechanisms, M. Shahani, ed., pp. 332-347, Elsevier, New York.

Paillard, J., P. Jordan, and M. Brouchon (1981) Visual motion cues in prismatic adaptation: Evidence of two separate and additive processes. Acta Psychol. 48: 253-270.

Perinen, M. T., and A. Vighetto (1983) Optic ataxia: A specific disorder in visuomotor coordination. In Spatially Oriented Behavior, A. Hein and M. Jeannerod, eds., pp. 305-326, Springer, New York.

Prablanc, C., J. E. Echallier, E. Komilis, and M. Jeannerod (1979a) Optimal response of eye and hand motor systems in pointing at a visual target. I. Spatio-temporal characteristics of eye and hand movements and their relationships when varying the amount of visual information. Biol. Cybernet. 35: 113-124.

Prablanc, C., J. E. Echallier, M. Jeannerod, and E. Komalis (1979b) Optimal response of the eye and hand motor systems in pointing at a visual target. II. Static and dynamic visual cues in the control of hand movement. Biol. Cybernet. 35: 183-187.

Priest, H. F., and J. E. Cutting (1985) Visual flow and direction of locomotion. Science 227: 1063-1065.

Regan, D., and K. Beverly (1982) How do we avoid confounding the direction we are looking and the direction we are moving? Science 215: 194-196.

Saito, H., M. Yukie, K. Tanaka, K. Hikosaka, Y. Fukada, and E. Iwai (1986) Integration of direction signals of image motion in the superior temporal sulcus of the macaque monkey. J. Neurosci. 6: 145-157.

Seal, J., and S. Commenges (1985) A quantitative analysis of stimulusand movement-related responses in the posterior parietal cortex of the monkey. Exp. Brain Res. 58: 144-153.

Seal, J., C. Gross, and B. Bioulac (1982) Activity of neurons in area 5 during a simple arm movement in monkeys before and after deafferentation of the trained limb. Brain Res. 250: 229-243.

Soechting, J. F. and F. Lacquaniti (1981) Invariant characteristics of a pointing movement in response to a change in target location. $\mathrm{J}$. Neurosci. 1: 710-720.

Tanaka, K., K. Hikosaka, H. Saito, M. Yukic, Y. Fukada, and E. Iwai (1986) Analysis of local and wide-field movements in the superior temporal visual areas of the macaque monkey. J. Neurosci. 6: 134144. 